\title{
Nuclear magnetic shielding constants of Dirac one-electron atoms in some low-lying discrete energy eigenstates
}

\author{
Patrycja Stefańska* \\ Atomic and Optical Physics Division, Department of Atomic, Molecular and Optical Physics, \\ Faculty of Applied Physics and Mathematics, Gdansk University of Technology, \\ Narutowicza 11/12, 80-233 Gdańsk, Poland
}

\section{Abstract}

We present tabulated data for the nuclear magnetic shielding constants $(\sigma)$ of the Dirac one-electron atoms with a pointlike, motionless and spinless nucleus of charge $Z e$. Utilizing the exact general analytical formula for $\sigma$ derived by us [P. Stefańska, Phys. Rev. A. 94 (2016) 012508/1-15], valid for an arbitrary discrete energy eigenstate, we have computed the numerical values of the magnetic shielding factors for the ground state and for the first and the second set of excited states, i.e.: $2 \mathrm{~s}_{1 / 2}, 2 \mathrm{p}_{1 / 2}, 2 \mathrm{p}_{3 / 2}, 3 \mathrm{~s}_{1 / 2}, 3 \mathrm{p}_{1 / 2}, 3 \mathrm{p}_{3 / 2}, 3 \mathrm{~d}_{3 / 2}$, and $3 \mathrm{~d}_{5 / 2}$, of the relativistic hydrogenic ions with the nuclear charge numbers from the range $1 \leqslant Z \leqslant 137$. The comparisons of our results with the numerical values reported by other authors for some atomic states are also presented.

Keywords: Hydrogenlike atom, Shielding constant, Magnetic field, Screening factor, Nuclear magnetic resonance

Published as: At. Data Nucl. Data Tables 120 (2018) 352-372

doi: $10.1016 /$ j.adt.2017.05.005

${ }^{*}$ Corresponding author.

Email address: pstefanska@mif.pg.gda.pl (Patrycja Stefańska) 


\section{Contents}

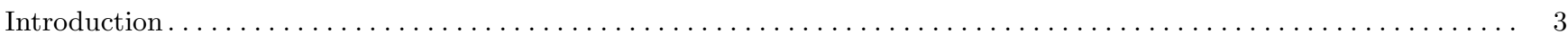

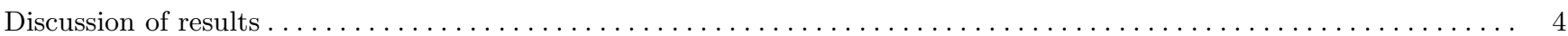

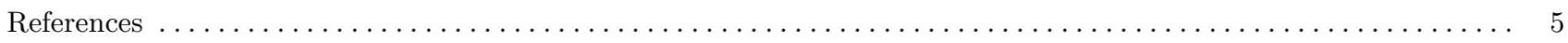

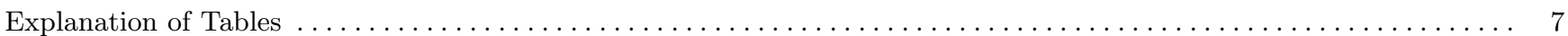

Tables

1. Magnetic shielding constants $\sigma$ (in units of $\alpha^{2}$ ) for the ground state $\left(1 \mathrm{~s}_{1 / 2}\right)$ of Dirac one-electron atoms. The number in brackets is the power of 10 by which the corresponding entry is to be multiplied. The value of the inverse of the fine-structure constant used was $\alpha^{-1}=137.035999139$ (from CODATA 2014) . . . . . . . 8

2. Magnetic shielding constants $\sigma$ (in units of $\alpha^{2}$ ) of Dirac one-electron atoms in the excited state $2 \mathrm{~s}_{1 / 2}$. The number in brackets is the power of 10 by which the corresponding entry is to be multiplied. The value of the inverse of the fine-structure constant used was $\alpha^{-1}=137.035999139$ (from CODATA 2014). . . . . . . 9

3. Magnetic shielding constants $\sigma$ (in units of $\alpha^{2}$ ) of Dirac one-electron atoms in the excited state $2 \mathrm{p}_{1 / 2}$. The number in brackets is the power of 10 by which the corresponding entry is to be multiplied. The value of the inverse of the fine-structure constant used was $\alpha^{-1}=137.035999139$ (from CODATA 2014). . . . . . . . 10

4. Magnetic shielding constants $\sigma$ (in units of $\alpha^{2}$ ) of Dirac one-electron atoms in the excited state $2 \mathrm{p}_{3 / 2}(\mu= \pm 1 / 2$ ). The number in brackets is the power of 10 by which the corresponding entry is to be multiplied. The value of the inverse of the fine-structure constant used was $\alpha^{-1}=137.035999139$ (from CODATA 2014). . . . . . 11

5. Magnetic shielding constants $\sigma$ (in units of $\alpha^{2}$ ) of Dirac one-electron atoms in the excited state $2 \mathrm{p}_{3 / 2}(\mu= \pm 3 / 2$ ). The number in brackets is the power of 10 by which the corresponding entry is to be multiplied. The value of the inverse of the fine-structure constant used was $\alpha^{-1}=137.035999139$ (from CODATA 2014) . . . . . . 12

6. Magnetic shielding constants $\sigma$ (in units of $\alpha^{2}$ ) of Dirac one-electron atoms in the excited state $3 \mathrm{~s}_{1 / 2}$. The number in brackets is the power of 10 by which the corresponding entry is to be multiplied. The value of the inverse of the fine-structure constant used was $\alpha^{-1}=137.035999139$ (from CODATA 2014). . . . . . . 13

7. Magnetic shielding constants $\sigma$ (in units of $\alpha^{2}$ ) of Dirac one-electron atoms in the excited state $3 \mathrm{p}_{1 / 2}$. The number in brackets is the power of 10 by which the corresponding entry is to be multiplied. The value of the inverse of the fine-structure constant used was $\alpha^{-1}=137.035999139$ (from CODATA 2014). . . . . . . 14

8. Magnetic shielding constants $\sigma$ (in units of $\alpha^{2}$ ) of Dirac one-electron atoms in the excited state $3 \mathrm{p}_{3 / 2}(\mu= \pm 1 / 2$ ). The number in brackets is the power of 10 by which the corresponding entry is to be multiplied. The value of the inverse of the fine-structure constant used was $\alpha^{-1}=137.035999139$ (from CODATA 2014). . . . . . 15

9. Magnetic shielding constants $\sigma$ (in units of $\alpha^{2}$ ) of Dirac one-electron atoms in the excited state $3 \mathrm{p}_{3 / 2}(\mu= \pm 3 / 2$ ). The number in brackets is the power of 10 by which the corresponding entry is to be multiplied. The value of the inverse of the fine-structure constant used was $\alpha^{-1}=137.035999139$ (from CODATA 2014) . . . . . . 16

10. Magnetic shielding constants $\sigma$ (in units of $\alpha^{2}$ ) of Dirac one-electron atoms in the excited state $3 \mathrm{~d}_{3 / 2}(\mu= \pm 1 / 2$ ). The number in brackets is the power of 10 by which the corresponding entry is to be multiplied. The value of

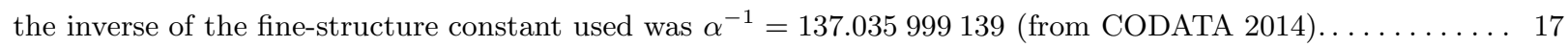
Magnetic shielding constants $\sigma$ (in units of $\alpha^{2}$ ) of Dirac one-electron atoms in the excited state $3 \mathrm{~d}_{3 / 2}(\mu= \pm 3 / 2$ ). The number in brackets is the power of 10 by which the corresponding entry is to be multiplied. The value of the inverse of the fine-structure constant used was $\alpha^{-1}=137.035999139$ (from CODATA 2014) . . . . . . 18 
12. Magnetic shielding constants $\sigma$ (in units of $\alpha^{2}$ ) of Dirac one-electron atoms in the excited state $3 \mathrm{~d}_{5 / 2}(\mu= \pm 1 / 2)$. The number in brackets is the power of 10 by which the corresponding entry is to be multiplied. The value of the inverse of the fine-structure constant used was $\alpha^{-1}=137.035999139$ (from CODATA 2014) . . . . . . 19

13. Magnetic shielding constants $\sigma$ (in units of $\alpha^{2}$ ) of Dirac one-electron atoms in the excited state $3 \mathrm{~d}_{5 / 2}(\mu= \pm 3 / 2)$. The number in brackets is the power of 10 by which the corresponding entry is to be multiplied. The value of the inverse of the fine-structure constant used was $\alpha^{-1}=137.035999139$ (from CODATA 2014). . . . . 20

14. Magnetic shielding constants $\sigma$ (in units of $\alpha^{2}$ ) of Dirac one-electron atoms in the excited state $3 \mathrm{~d}_{3 / 2}(\mu= \pm 5 / 2)$. The number in brackets is the power of 10 by which the corresponding entry is to be multiplied. The value of the inverse of the fine-structure constant used was $\alpha^{-1}=137.035999139$ (from CODATA 2014)........ 21

15. Comparison of present values (the upper entries) of the magnetic shielding constants $\sigma$ (in units of $\alpha^{2}$ ) for selected Dirac one-electron atoms in the states with zero radial quantum number, i.e: $1 \mathrm{~s}_{1 / 2}, 2 \mathrm{p}_{3 / 2}$, and $3 \mathrm{~d}_{5 / 2}$, with those reported by Moore [22] (the lower entries). All the numbers were obtained using $\alpha^{-1}=137.0359895$ (from CODATA 1986). The present results were computed from the analytical formula given by Eqs. (1)-(4). . . . 22

16. Comparison of present values (the upper entries) of the magnetic shielding constants $\sigma$ (in units of $10^{-6}$ ) for selected Dirac one-electron atoms in the ground state $\left(1 \mathrm{~s}_{1 / 2}\right)$ and the first excited state, i.e: $2 \mathrm{p}_{1 / 2}$ and $2 \mathrm{p}_{3 / 2}$, with those obtained by Pyper and Zhang [23] (the lower entries). All the numbers were obtained using $\alpha^{-1}=$ 137.035 9895 (from CODATA 1986). The present results were computed from the analytical formula given by

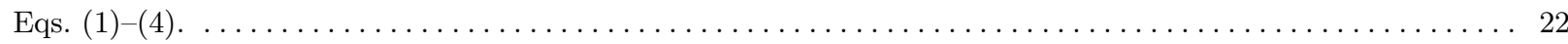

\section{Introduction}

When an atom or a molecule is placed in an external magnetic field, then electrons circling around their nuclei will begin to interact with the perturbing field. In this way, an additional magnetic field, oriented opposite to the external field, is generated in the system. Consequently, the strength of the magnetic field "effectively sensed" by the nucleus decreases. This phenomenon is called nuclear magnetic shielding. The changes that occur in the location of the nucleus can be fully characterized by the magnetic shielding constant (or the nuclear screening factor). This physical quantity - of course, depending on the electron density - is directly related to the chemical shift, by which the position of the signal in the NMR (Nuclear Magnetic Resonance) spectrum is determined. This makes the magnetic shielding constant $(\sigma)$ one of the most important atomic parameters currently used in chemistry and medicine.

There are many experimental results for the magnetic screening constants for many-electron atoms and molecules. But relativistic theoretical investigations of that quantity for such complex systems are quite complicated - very often they come down to performing numerical calculations, which almost always require access to specialized software. The numerical values for $\sigma$ for some closed-shell atoms and ions were reported, among others, in Refs. [1-3]. However, for the simplest systems, like one-electron atoms, the purely analytical relativistic calculations for the magnetic shielding constant (and also for another properties of the atom) are possible, and this was suggested already in 1969 by Hegstrom in Ref. [4]. One of such a technique which allows one to derive analytically the formula for the shielding factor $(\sigma)$ is the one based on the perturbation theory combined with the Sturmian expansion of the first-order generalized Dirac-Coulomb 
Green function, proposed in Ref. [5]. Using the method presented in that article, some time ago we have found the closed-form expression for $\sigma$ for the ground state of relativistic hydrogenlike atom [6], in agreement with corresponding formulas derived earlier by other authors in completely different ways. (For other applications of the technique proposed by Szmytkowski in Ref. [5] to some electromagnetic properties of the ground state of Dirac one-electron atoms, see Refs. [7-15].).

Quite recently, we have shown that the usefulness of the aforementioned method goes beyond the study of the atomic ground state. In Refs. [16-20] we have considered an arbitrary discrete energy eigenstate of relativistic hydrogenlike atom, in order to obtain analytical expressions (and further - the numerical values) for some properties illustrating the influence of the external electromagnetic perturbations on the atom. In particular, in Ref. [16] one can find a detailed derivation of the formula for the magnetic shielding constant of Dirac one-electron atom being in an arbitrary energy eigenstate. The quantum state of the electron is generally characterized by the set of quantum numbers $\{n, \kappa, \mu\}$, in which $n$ denotes the radial quantum number, the Dirac quantum number $\kappa$ is an integer different form zero, whereas $\mu=-|\kappa|+\frac{1}{2},-|\kappa|+\frac{3}{2}, \ldots,|\kappa|-\frac{1}{2}$ is the magnetic quantum number. The final closed-form expression for the shielding factor for such a state we have arrived at in Ref. [16] reads as follows:

$$
\begin{array}{r}
\sigma \equiv \sigma_{n \kappa \mu}=\frac{\alpha^{2} Z}{N_{n \kappa}^{2}\left(4 \kappa^{2}-1\right)}\left[\kappa^{2}-\frac{\eta_{n \kappa}^{(+)}}{4}-\frac{\eta_{n \kappa}^{(-)}}{4}+\frac{\mu^{2}}{4 \kappa^{2}-1}\left(\frac{2 \kappa+1}{2 \kappa-1} \eta_{n \kappa}^{(+)}+\frac{2 \kappa-1}{2 \kappa+1} \eta_{n \kappa}^{(-)}\right)\right. \\
\left.+\frac{4 \kappa^{2} \mu^{2}}{4 \kappa^{2}-1}\left(\frac{4 \kappa^{2}-5}{4 \kappa^{2}-1}+\frac{8 \kappa\left[2 \kappa\left(n+\gamma_{\kappa}\right)-N_{n \kappa}\right](\alpha Z)^{2}}{\gamma_{\kappa}\left(4 \gamma_{\kappa}^{2}-1\right) N_{n \kappa}^{2}}\right)\right],
\end{array}
$$

where

$$
\eta_{n \kappa}^{( \pm)}=\frac{(2 \kappa \pm 1) N_{n \kappa}}{n+\gamma_{\kappa} \pm N_{n \kappa}}
$$

with

$$
N_{n \kappa}=\sqrt{n^{2}+2 n \gamma_{\kappa}+\kappa^{2}}
$$

and

$$
\gamma_{\kappa}=\sqrt{\kappa^{2}-(\alpha Z)^{2}}
$$

In Ref. [16] we have proved analytically that the above result is valid for an arbitrary atomic state. However, in the aforementioned article, there are no tables with the numerical values of the relativistic shielding factors of hydrogenic ions. This kind of data may be useful - among others - to those who deal with the spectroscopic methods, for example, the NMR technique. This fact, as well as the lack of sufficiently large data sets in the literature, prompted us to carry out numerical calculations for the magnetic shielding constant for one-electron atoms ions with the nuclear charge numbers from the whole range $1 \leqslant Z \leqslant 137$. Our results will be discussed briefly in the next section.

\section{Discussion of results}

Numerical results presented in this work has been computed with the help of the exact analytical formula given in Eqs. (1)-(4). In Table 1 we have included the values of the magnetic shielding factors for the ground state $\left(1 \mathrm{~s}_{1 / 2}\right)$ of Dirac one-electron atoms. Tables $2-5$ contain the results for states belonging to the first set of excited state, i.e.: $2 \mathrm{~s}_{1 / 2}$, $2 \mathrm{p}_{1 / 2}$, and $2 \mathrm{p}_{3 / 2}$. The second excited atomic states (i.e.: $3 \mathrm{~s}_{1 / 2}, 3 \mathrm{p}_{1 / 2}, 3 \mathrm{p}_{3 / 2}, 3 \mathrm{~d}_{3 / 2}$, and $3 \mathrm{~d}_{5 / 2}$ ) are presented in Tables 
6-14. For the excited states, all possible values of the magnetic quantum number $\mu$ were considered. The Reader should observe that for the states with $\kappa= \pm 1$, there is a restriction for the nuclear charge number, i.e.: $Z<\alpha^{-1} \frac{\sqrt{3}}{2} \simeq 118.67$ (see Tables 1-3, 6, and 7); a detailed explanation of this limitation can be found just follow Eq. (3.17) in Ref. [16].

The value of the inverse of the fine-structure constant we have used during creating Tables $1-14$ was $\alpha^{-1}=$ 137.035 999 139, and was taken from the newest CODATA 2014 report on Recommended Values of the Fundamental Physical Constants [21]. However, in order to make the most accurate comparison of our exact results with the numerical results reported earlier by other authors for some atomic states [22, 23], we have performed two additional tables with the values of $\sigma$ obtained using $\alpha^{-1}=137.0359895$ (from CODATA 1986) [24].

States with zero radial quantum number $n=0$ have been considered in Table 15 , where we have confronted our results with the values published by Moore in Ref. [22]. Actually, to be able to compare these numbers, we had to add the corresponding numerical components provided by her for a given atomic state. The resulting sums of the three ingredients for each state (the lower entries in Table 15) appear to be in a pretty good agreement with our exact results (the upper entries therein).

In contrast to the values of the screening constants presented in Tables 1-15 (where they were given in units of $\alpha^{2}$ ), the numbers included in Table 16 should be multiplied only by the commonly used factor of $10^{-6}$. We have rescaled in this way a part of the data from Tables 1 and $3-5$ (i.e. for states $1 \mathrm{~s}_{1 / 2}, 2 \mathrm{p}_{1 / 2}$, and $2 \mathrm{p}_{3 / 2}$ ), because we wanted to compare them with the corresponding numerical values provided by Pyper and Zhang [23]. The agreement between their results and ours is also very good, and this has been shown in Table 16. The value of the magnetic shielding factor for the ground state of relativistic hydrogen atom $(Z=1)$ can be found - among others - in the paper by Feiock and Johnson [25]. Their result, which is $\sigma=17.75 \times 10^{-6}$ (not shown in Table 16), also agrees with our result for this case.

\section{Acknowledgments}

I am indebted to Professor A. Rutkowski for his suggestion to publish present results. I also thank Professor R. Szmytkowski for technical assistance in carrying out the calculations.

\section{References}

\section{References}

[1] A. Dalgarno, Advances in Physics 11 (1962) 281.

[2] D. Kolb, W. R. Johnson, P. Shorer, Physical Review A 26 (1982) 19.

[3] W. R. Johnson, D. Kolb, K. N. Huang, Atomic Data and Nuclear Data Tables 28 (1983) 333.

[4] R. A. Hegstrom, Physical Review A 184 (1969) 17.

[5] R. Szmytkowski, Journal of Physics B 30 (1997) 825 [erratum: Journal of Physics B 30 (1997) 2747; addendum: arXiv:physics/9902050].

[6] P. Stefańska, R. Szmytkowski, International Journal of Quantum Chemistry 112 (2012) 1363.

[7] R. Szmytkowski, Journal of Physics B 35 (2002) 1379. 
[8] R. Szmytkowski, K. Mielewczyk, Journal of Physics B 37 (2004) 3961.

[9] R. Szmytkowski, Physical Review A 65 (2002) 012503.

[10] K. Mielewczyk, R. Szmytkowski, Physical Review A 73 (2006) 022511 [erratum: Physical Review A 73 (2006) 039908].

[11] R. Szmytkowski, P. Stefańska, e-print arXiv:1102.1811.

[12] R. Szmytkowski, P. Stefańska, Physical Review A 85 (2012) 042502.

[13] R. Szmytkowski, P. Stefańska, Physical Review A 89 (2014) 012501.

[14] R. Szmytkowski, G. Łukasik, Physical Review A 93 (2016) 062502.

[15] R. Szmytkowski, G. Łukasik, Atomic Data and Nuclear Data Tables 111-112 (2016) 41.

[16] P. Stefańska, Physical Review A 94 (2016) 012508.

[17] P. Stefańska, Physical Review A 92 (2015) 032504.

[18] P. Stefańska, Physical Review A 93 (2016) 022504.

[19] P. Stefańska, Atomic Data and Nuclear Data Tables 108 (2016) 193.

[20] P. Stefańska, Atomic Data and Nuclear Data Tables 113 (2017) 316.

[21] P. J. Mohr, D. B. Newell, B. N. Taylor, CODATA Recommended Values of the Fundamental Physical Constants: 2014, arXiv:abs/1507.07956.

[22] E. A. Moore, Molecular Physics 97 (1999) 375.

[23] N. C. Pyper, Z. C. Zhang, Molecular Physics 97 (1999) 391.

[24] E. R. Cohen, B. N. Taylor, Reviews of Modern Physics 59 (1987) 1121.

[25] F. D. Feiock, W. R. Johnson, Physical Review A 187 (1969) 39. 


\section{Explanation of Tables}

In all the tables we have used the following notation:

$\begin{array}{ll}\sigma & \text { Magnetic dipole shielding constant } \\ Z & \text { Nuclear charge number } \\ \alpha & \text { Fine structure constant } \\ \mu & \text { Magnetic quantum number; } \mu=-j, \ldots, j\end{array}$

The atomic states are referred to as $\mathcal{N} x_{j}$, according to the following guidelines:

$\mathcal{N} \quad$ Principal quantum number

$\mathcal{N}=n+|\kappa| \quad$ Note that for the radial quantum number $n=0, \kappa$ is the negative integer!

$\kappa \quad$ Dirac quantum number; $\kappa=(l-j)(2 j+1)$

$j \quad$ Total angular momentum quantum number; $j=|\kappa|-\frac{1}{2}=l \pm \frac{1}{2}$

$x \equiv s \quad$ States with the orbital angular momentum quantum number $l=0$

$x \equiv p \quad$ States with the orbital angular momentum quantum number $l=1$

$x \equiv d \quad$ States with the orbital angular momentum quantum number $l=2$ 
Table 1

Magnetic shielding constants $\sigma$ (in units of $\left.\alpha^{2}\right)$ for the ground state $\left(1 \mathrm{~s}_{1 / 2}\right)$ of Dirac one-electron atoms. The number in brackets is the power of 10 by which the corresponding entry is to be multiplied. The value of the inverse of the fine-structure constant used was $\alpha^{-1}=137.035999139$ (from CODATA 2014).

\begin{tabular}{|c|c|c|c|c|c|}
\hline$Z$ & $\sigma\left[\alpha^{2}\right]$ & $Z$ & $\sigma\left[\alpha^{2}\right]$ & $Z$ & $\sigma\left[\alpha^{2}\right]$ \\
\hline 1 & $3.333811647331(-1)$ & 41 & $1.746602974210(+1)$ & 81 & $7.817869668184(+1)$ \\
\hline 2 & $6.670494089568(-1)$ & 42 & $1.811496081836(+1)$ & 82 & $8.174063737041(+1)$ \\
\hline 3 & $1.001292267930(+0)$ & 43 & $1.878357035573(+1)$ & 83 & $8.552930762593(+1)$ \\
\hline 4 & $1.336398189894(+0)$ & 44 & $1.947283571199(+1)$ & 84 & $8.956539142795(+1)$ \\
\hline 5 & $1.672656996173(+0)$ & 45 & $2.018378683624(+1)$ & 85 & $9.387209193087(+1)$ \\
\hline 6 & $2.010360345643(+0)$ & 46 & $2.091751011580(+1)$ & 86 & $9.847552267330(+1)$ \\
\hline 7 & $2.349802205046(+0)$ & 47 & $2.167515254880(+1)$ & 87 & $1.034051735170(+2)$ \\
\hline 8 & $2.691279326667(+0)$ & 48 & $2.245792627529(+1)$ & 88 & $1.086944684530(+2)$ \\
\hline 9 & $3.035091734955(+0)$ & 49 & $2.326711350387(+1)$ & 89 & $1.143814370449(+2)$ \\
\hline 10 & $3.381543223662(+0)$ & 50 & $2.410407187488(+1)$ & 90 & $1.205095273710(+2)$ \\
\hline 11 & $3.730941865202(+0)$ & 51 & $2.497024030612(+1)$ & 91 & $1.271285963977(+2)$ \\
\hline 12 & $4.083600533933(+0)$ & 52 & $2.586714537291(+1)$ & 92 & $1.342961245207(+2)$ \\
\hline 13 & $4.439837445187(+0)$ & 53 & $2.679640828022(+1)$ & 93 & $1.420787155992(+2)$ \\
\hline 14 & $4.799976711934(+0)$ & 54 & $2.775975249240(+1)$ & 94 & $1.505539637316(+2)$ \\
\hline 15 & $5.164348921084(+0)$ & 55 & $2.875901209373(+1)$ & 95 & $1.598127955178(+2)$ \\
\hline 16 & $5.533291731519(+0)$ & 56 & $2.979614096288(+1)$ & 96 & $1.699624349813(+2)$ \\
\hline 17 & $5.907150496098(+0)$ & 57 & $3.087322285525(+1)$ & 97 & $1.811301927131(+2)$ \\
\hline 18 & $6.286278909991(+0)$ & 58 & $3.199248249922(+1)$ & 98 & $1.934683588841(+2)$ \\
\hline 19 & $6.671039687874(+0)$ & 59 & $3.315629782747(+1)$ & 99 & $2.071605935952(+2)$ \\
\hline 20 & $7.061805272665(+0)$ & 60 & $3.436721348028(+1)$ & 100 & $2.224303767330(+2)$ \\
\hline 21 & $7.458958578693(+0)$ & 61 & $3.562795573774(+1)$ & 101 & $2.395523341337(+2)$ \\
\hline 22 & $7.862893772380(+0)$ & 62 & $3.694144905961(+1)$ & 102 & $2.588676489615(+2)$ \\
\hline 23 & $8.274017093771(+0)$ & 63 & $3.831083443758(+1)$ & 103 & $2.808053844970(+2)$ \\
\hline 24 & $8.692747722474(+0)$ & 64 & $3.973948979521(+1)$ & 104 & $3.059125404493(+2)$ \\
\hline 25 & $9.119518691881(+0)$ & 65 & $4.123105270560(+1)$ & 105 & $3.348973163289(+2)$ \\
\hline 26 & $9.554777855843(+0)$ & 66 & $4.278944573894(+1)$ & 106 & $3.686928794988(+2)$ \\
\hline 27 & $9.998988912301(+0)$ & 67 & $4.441890480008(+1)$ & 107 & $4.085539365807(+2)$ \\
\hline 28 & $1.045263248880(+1)$ & 68 & $4.612401087408(+1)$ & 108 & $4.562076240559(+2)$ \\
\hline 29 & $1.091620729515(+1)$ & 69 & $4.790972566522(+1)$ & 109 & $5.140980242816(+2)$ \\
\hline 30 & $1.139023134915(+1)$ & 70 & $4.978143169555(+1)$ & 110 & $5.857998577753(+2)$ \\
\hline 31 & $1.187524328142(+1)$ & 71 & $5.174497752454(+1)$ & 111 & $6.767556412750(+2)$ \\
\hline 32 & $1.237180372659(+1)$ & 72 & $5.380672886556(+1)$ & 112 & $7.956754800431(+2)$ \\
\hline 33 & $1.288049680794(+1)$ & 73 & $5.597362651196(+1)$ & 113 & $9.574166598994(+2)$ \\
\hline 34 & $1.340193172405(+1)$ & 74 & $5.825325214966(+1)$ & 114 & $1.189558089718(+3)$ \\
\hline 35 & $1.393674444622(+1)$ & 75 & $6.065390333183(+1)$ & 115 & $1.549704391322(+3)$ \\
\hline 36 & $1.448559953664(+1)$ & 76 & $6.318467913180(+1)$ & 116 & $2.181580134554(+3)$ \\
\hline 37 & $1.504919209807(+1)$ & 77 & $6.585557828282(+1)$ & 117 & $3.571862766845(+3)$ \\
\hline 38 & $1.562824986692(+1)$ & 78 & $6.867761197138(+1)$ & 118 & $9.084394854718(+3)$ \\
\hline 39 & $1.622353546292(+1)$ & 79 & $7.166293388930(+1)$ & & \\
\hline 40 & $1.683584880967(+1)$ & 80 & $7.482499069208(+1)$ & & \\
\hline
\end{tabular}


Table 2

Magnetic shielding constants $\sigma$ (in units of $\alpha^{2}$ ) of Dirac one-electron atoms in the excited state $2 \mathrm{~s}_{1 / 2}$. The number in brackets is the power of 10 by which the corresponding entry is to be multiplied. The value of the inverse of the fine-structure constant used was $\alpha^{-1}=137.035999139$ (from CODATA 2014).

\begin{tabular}{|c|c|c|c|c|c|}
\hline$Z$ & $\sigma\left[\alpha^{2}\right]$ & $Z$ & $\sigma\left[\alpha^{2}\right]$ & $Z$ & $\sigma\left[\alpha^{2}\right]$ \\
\hline 1 & $8.334039107064(-2)$ & 41 & $3.997600222359(+0)$ & 81 & $1.571351017454(+1)$ \\
\hline 2 & $1.667231453748(-1)$ & 42 & $4.130373857431(+0)$ & 82 & $1.641605092176(+1)$ \\
\hline 3 & $2.501907102596(-1)$ & 43 & $4.266391434659(+0)$ & 83 & $1.716707475740(+1)$ \\
\hline 4 & $3.337857016011(-1)$ & 44 & $4.405831332584(+0)$ & 84 & $1.797140369287(+1)$ \\
\hline 5 & $4.175509888277(-1)$ & 45 & $4.548882753481(+0)$ & 85 & $1.883447735330(+1)$ \\
\hline 6 & $5.015297808566(-1)$ & 46 & $4.695746564523(+0)$ & 86 & $1.976245167495(+1)$ \\
\hline 7 & $5.857657132582(-1)$ & 47 & $4.846636213933(+0)$ & 87 & $2.076231677664(+1)$ \\
\hline 8 & $6.703029369684(-1)$ & 48 & $5.001778729970(+0)$ & 88 & $2.184203844590(+1)$ \\
\hline 9 & $7.551862088762(-1)$ & 49 & $5.161415811537(+0)$ & 89 & $2.301072888914(+1)$ \\
\hline 10 & $8.404609846285(-1)$ & 50 & $5.325805020255(+0)$ & 90 & $2.427885398357(+1)$ \\
\hline 11 & $9.261735140035(-1)$ & 51 & $5.495221085057(+0)$ & 91 & $2.565848637678(+1)$ \\
\hline 12 & $1.012370939221(+0)$ & 52 & $5.669957331700(+0)$ & 92 & $2.716361660258(+1)$ \\
\hline 13 & $1.099101396575(+0)$ & 53 & $5.850327251216(+0)$ & 93 & $2.881053819696(+1)$ \\
\hline 14 & $1.186414121797(+0)$ & 54 & $6.036666223042(+0)$ & 94 & $3.061832801062(+1)$ \\
\hline 15 & $1.274359559565(+0)$ & 55 & $6.229333410669(+0)$ & 95 & $3.260945011467(+1)$ \\
\hline 16 & $1.362989477637(+0)$ & 56 & $6.428713849971(+0)$ & 96 & $3.481052176147(+1)$ \\
\hline 17 & $1.452357086056(+0)$ & 57 & $6.635220753092(+0)$ & 97 & $3.725329411875(+1)$ \\
\hline 18 & $1.542517161970(+0)$ & 58 & $6.849298053857(+0)$ & 98 & $3.997592096977(+1)$ \\
\hline 19 & $1.633526180598(+0)$ & 59 & $7.071423224280(+0)$ & 99 & $4.302461842982(+1)$ \\
\hline 20 & $1.725442452936(+0)$ & 60 & $7.302110395887(+0)$ & 100 & $4.645586300011(+1)$ \\
\hline 21 & $1.818326270827(+0)$ & 61 & $7.541913824393(+0)$ & 101 & $5.033934212128(+1)$ \\
\hline 22 & $1.912240060086(+0)$ & 62 & $7.791431741840(+0)$ & 102 & $5.476197434780(+1)$ \\
\hline 23 & $2.007248542403(+0)$ & 63 & $8.051310646855(+0)$ & 103 & $5.983347838958(+1)$ \\
\hline 24 & $2.103418906810(+0)$ & 64 & $8.322250091266(+0)$ & 104 & $6.569423189190(+1)$ \\
\hline 25 & $2.200820991594(+0)$ & 65 & $8.605008030248(+0)$ & 105 & $7.252659472489(+1)$ \\
\hline 26 & $2.299527477552(+0)$ & 66 & $8.900406813608(+0)$ & 106 & $8.057161366631(+1)$ \\
\hline 27 & $2.399614093624(+0)$ & 67 & $9.209339908149(+0)$ & 107 & $9.015433969588(+1)$ \\
\hline 28 & $2.501159835993(+0)$ & 68 & $9.532779455591(+0)$ & 108 & $1.017234117123(+2)$ \\
\hline 29 & $2.604247201842(+0)$ & 69 & $9.871784787734(+0)$ & 109 & $1.159152367476(+2)$ \\
\hline 30 & $2.708962439094(+0)$ & 70 & $1.022751204100(+1)$ & 110 & $1.336626242678(+2)$ \\
\hline 31 & $2.815395813536(+0)$ & 71 & $1.060122503690(+1)$ & 111 & $1.563884307016(+2)$ \\
\hline 32 & $2.923641894917(+0)$ & 72 & $1.099430762400(+1)$ & 112 & $1.863733718839(+2)$ \\
\hline 33 & $3.033799863705(+0)$ & 73 & $1.140827771213(+1)$ & 113 & $2.275128203952(+2)$ \\
\hline 34 & $3.145973840398(+0)$ & 74 & $1.184480327146(+1)$ & 114 & $2.870448942588(+2)$ \\
\hline 35 & $3.260273239442(+0)$ & 75 & $1.230572062005(+1)$ & 115 & $3.800992106755(+2)$ \\
\hline 36 & $3.376813150031(+0)$ & 76 & $1.279305538535(+1)$ & 116 & $5.444431551858(+2)$ \\
\hline 37 & $3.495714746272(+0)$ & 77 & $1.330904660023(+1)$ & 117 & $9.080005674542(+2)$ \\
\hline 38 & $3.617105729484(+0)$ & 78 & $1.385617448619(+1)$ & 118 & $2.355074407820(+3)$ \\
\hline 39 & $3.741120805656(+0)$ & 79 & $1.443719258992(+1)$ & & \\
\hline 40 & $3.867902201437(+0)$ & 80 & $1.505516507875(+1)$ & & \\
\hline
\end{tabular}


Table 3

Magnetic shielding constants $\sigma$ (in units of $\alpha^{2}$ ) of Dirac one-electron atoms in the excited state $2 \mathrm{p}_{1 / 2}$. The number in brackets is the power of 10 by which the corresponding entry is to be multiplied. The value of the inverse of the fine-structure constant used was $\alpha^{-1}=137.035999139$ (from CODATA 2014).

\begin{tabular}{|c|c|c|c|c|c|}
\hline$Z$ & $\sigma\left[\alpha^{2}\right]$ & $Z$ & $\sigma\left[\alpha^{2}\right]$ & $Z$ & $\sigma\left[\alpha^{2}\right]$ \\
\hline 1 & $2.782118900586(+3)$ & 41 & $7.073605130669(+1)$ & 81 & $4.268987601923(+1)$ \\
\hline 2 & $1.391156693555(+3)$ & 42 & $6.920391087899(+1)$ & 82 & $4.253568075818(+1)$ \\
\hline 3 & $9.275458812054(+2)$ & 43 & $6.774808572768(+1)$ & 83 & $4.240611438939(+1)$ \\
\hline 4 & $6.957729597111(+2)$ & 44 & $6.636351171 \quad 133(+1)$ & 84 & $4.230218908270(+1)$ \\
\hline 5 & $5.567352419207(+2)$ & 45 & $6.504558340066(+1)$ & 85 & $4.222510517120(+1)$ \\
\hline 6 & $4.640651768925(+2)$ & 46 & $6.379010482398(+1)$ & 86 & $4.217627714061(+1)$ \\
\hline 7 & $3.978909669943(+2)$ & 47 & $6.259324655342(+1)$ & 87 & $4.215736488486(+1)$ \\
\hline 8 & $3.482767222004(+2)$ & 48 & $6.145150821268(+1)$ & 88 & $4.217031142058(+1)$ \\
\hline 9 & $3.097025115434(+2)$ & 49 & $6.036168563751(+1)$ & 89 & $4.221738857880(+1)$ \\
\hline 10 & $2.788563837135(+2)$ & 50 & $5.932084204416(+1)$ & 90 & $4.230125261997(+1)$ \\
\hline 11 & $2.536307400749(+2)$ & 51 & $5.832628266254(+1)$ & 91 & $4.242501228544(+1)$ \\
\hline 12 & $2.326205211906(+2)$ & 52 & $5.737553237555(+1)$ & 92 & $4.259231255729(+1)$ \\
\hline 13 & $2.148529995819(+2)$ & 53 & $5.646631597617(+1)$ & 93 & $4.280743842405(+1)$ \\
\hline 14 & $1.996333756060(+2)$ & 54 & $5.559654071244(+1)$ & 94 & $4.307544434970(+1)$ \\
\hline 15 & $1.864521349974(+2)$ & 55 & $5.476428083960(+1)$ & 95 & $4.340231707680(+1)$ \\
\hline 16 & $1.749271473459(+2)$ & 56 & $5.396776394033(+1)$ & 96 & $4.379518209652(+1)$ \\
\hline 17 & $1.647662004198(+2)$ & 57 & $5.320535880893(+1)$ & 97 & $4.426256794431(+1)$ \\
\hline 18 & $1.557420230630(+2)$ & 58 & $5.247556472517(+1)$ & 98 & $4.481474797271(+1)$ \\
\hline 19 & $1.476751956119(+2)$ & 59 & $5.177700196926(+1)$ & 99 & $4.546418726240(+1)$ \\
\hline 20 & $1.404221872018(+2)$ & 60 & $5.110840345139(+1)$ & 100 & $4.622613420492(+1)$ \\
\hline 21 & $1.338668110010(+2)$ & 61 & $5.046860734826(+1)$ & 101 & $4.711941421401(+1)$ \\
\hline 22 & $1.279140098492(+2)$ & 62 & $4.985655065612(+1)$ & 102 & $4.816751062641(+1)$ \\
\hline 23 & $1.224852630502(+2)$ & 63 & $4.927126358438(+1)$ & 103 & $4.940006131445(+1)$ \\
\hline 24 & $1.175151414807(+2)$ & 64 & $4.871186472732(+1)$ & 104 & $5.085496966089(+1)$ \\
\hline 25 & $1.129486894895(+2)$ & 65 & $4.817755696354(+1)$ & 105 & $5.258144484154(+1)$ \\
\hline 26 & $1.087394109900(+2)$ & 66 & $4.766762404386(+1)$ & 106 & $5.464448525148(+1)$ \\
\hline 27 & $1.048477031051(+2)$ & 67 & $4.718142783913(+1)$ & 107 & $5.713167113854(+1)$ \\
\hline 28 & $1.012396254761(+2)$ & 68 & $4.671840622963(+1)$ & 108 & $6.016378170848(+1)$ \\
\hline 29 & $9.788592421701(+1)$ & 69 & $4.627807162767(+1)$ & 109 & $6.391200505719(+1)$ \\
\hline 30 & $9.476125109579(+1)$ & 70 & $4.586001013568(+1)$ & 110 & $6.862706229409(+1)$ \\
\hline 31 & $9.184353386252(+1)$ & 71 & $4.546388135254(+1)$ & 111 & $7.469111353803(+1)$ \\
\hline 32 & $8.911346466148(+1)$ & 72 & $4.508941885265(+1)$ & 112 & $8.271633642451(+1)$ \\
\hline 33 & $8.655408148122(+1)$ & 73 & $4.473643137479(+1)$ & 113 & $9.374773835554(+1)$ \\
\hline 34 & $8.415042348935(+1)$ & 74 & $4.440480477216(+1)$ & 114 & $1.097262326270(+2)$ \\
\hline 35 & $8.188924547711(+1)$ & 75 & $4.409450479113(+1)$ & 115 & $1.347075187532(+2)$ \\
\hline 36 & $7.975877992212(+1)$ & 76 & $4.380558076535(+1)$ & 116 & $1.788133909961(+2)$ \\
\hline 37 & $7.774853766263(+1)$ & 77 & $4.353817033378(+1)$ & 117 & $2.763233894940(+2)$ \\
\hline 38 & $7.584914007315(+1)$ & 78 & $4.329250531831(+1)$ & 118 & $6.641887213111(+2)$ \\
\hline 39 & $7.405217708967(+1)$ & 79 & $4.306891892840(+1)$ & & \\
\hline 40 & $7.235008656373(+1)$ & 80 & $4.286785449937(+1)$ & & \\
\hline
\end{tabular}


Table 4

Magnetic shielding constants $\sigma$ (in units of $\alpha^{2}$ ) of Dirac one-electron atoms in the excited state $2 \mathrm{p}_{3 / 2}(\mu= \pm 1 / 2)$. The number in brackets is the power of 10 by which the corresponding entry is to be multiplied. The value of the inverse of the fine-structure constant used was $\alpha^{-1}=137.035999139$ (from CODATA 2014).

\begin{tabular}{|c|c|c|c|c|c|}
\hline$Z$ & $\sigma\left[\alpha^{2}\right]$ & $Z$ & $\sigma\left[\alpha^{2}\right]$ & $Z$ & $\sigma\left[\alpha^{2}\right]$ \\
\hline 1 & $-2.781968897415(+3)$ & 47 & $-5.514965913733(+1)$ & 93 & $-2.165593616341(+1)$ \\
\hline 2 & $-1.390856668182(+3)$ & 48 & $-5.382867430870(+1)$ & 94 & $-2.124047243592(+1)$ \\
\hline 3 & $-9.270957955406(+2)$ & 49 & $-5.255790538763(+1)$ & 95 & $-2.083138472906(+1)$ \\
\hline 4 & $-6.951727565491(+2)$ & 50 & $-5.133432930687(+1)$ & 96 & $-2.042845685127(+1)$ \\
\hline 5 & $-5.559848448573(+2)$ & 51 & $-5.015515982506(+1)$ & 97 & $-2.003148109115(+1)$ \\
\hline 6 & $-4.631644902110(+2)$ & 52 & $-4.901782474997(+1)$ & 98 & $-1.964025777215(+1)$ \\
\hline 7 & $-3.968398755243(+2)$ & 53 & $-4.791994574013(+1)$ & 99 & $-1.925459483366(+1)$ \\
\hline 8 & $-3.470750911465(+2)$ & 54 & $-4.685932035049(+1)$ & 100 & $-1.887430743694(+1)$ \\
\hline 9 & $-3.083501862844(+2)$ & 55 & $-4.583390603666(+1)$ & 101 & $-1.849921759375(+1)$ \\
\hline 10 & $-2.773531895705(+2)$ & 56 & $-4.484180587267(+1)$ & 102 & $-1.812915381648(+1)$ \\
\hline 11 & $-2.519764820488(+2)$ & 57 & $-4.388125577208(+1)$ & 103 & $-1.776395078803(+1)$ \\
\hline 12 & $-2.308149836664(+2)$ & 58 & $-4.295061303080(+1)$ & 104 & $-1.740344905021(+1)$ \\
\hline 13 & $-2.128959460006(+2)$ & 59 & $-4.204834603487(+1)$ & 105 & $-1.704749470934(+1)$ \\
\hline 14 & $-1.975245481015(+2)$ & 60 & $-4.117302499730(+1)$ & 106 & $-1.669593915801(+1)$ \\
\hline 15 & $-1.841912539984(+2)$ & 61 & $-4.032331360582(+1)$ & 107 & $-1.634863881 \quad 172(+1)$ \\
\hline 16 & $-1.725139111401(+2)$ & 62 & $-3.949796147874(+1)$ & 108 & $-1.600545485961(+1)$ \\
\hline 17 & $-1.622002846802(+2)$ & 63 & $-3.869579733915(+1)$ & 109 & $-1.566625302816(+1)$ \\
\hline 18 & $-1.530230803335(+2)$ & 64 & $-3.791572282875(+1)$ & 110 & $-1.533090335718(+1)$ \\
\hline 19 & $-1.448028547513(+2)$ & 65 & $-3.715670689259(+1)$ & 111 & $-1.499927998718(+1)$ \\
\hline 20 & $-1.373960527835(+2)$ & 66 & $-3.641778067406(+1)$ & 112 & $-1.467126095735(+1)$ \\
\hline 21 & $-1.306864626665(+2)$ & 67 & $-3.569803286684(+1)$ & 113 & $-1.434672801357(+1)$ \\
\hline 22 & $-1.245790016129(+2)$ & 68 & $-3.499660547672(+1)$ & 114 & $-1.402556642568(+1)$ \\
\hline 23 & $-1.189951225530(+2)$ & 69 & $-3.431268995185(+1)$ & 115 & $-1.370766481354(+1)$ \\
\hline 24 & $-1.138693691896(+2)$ & 70 & $-3.364552364433(+1)$ & 116 & $-1.339291498115(+1)$ \\
\hline 25 & $-1.091467578399(+2)$ & 71 & $-3.299438657067(+1)$ & 117 & $-1.308121175841(+1)$ \\
\hline 26 & $-1.047807634676(+2)$ & 72 & $-3.235859844203(+1)$ & 118 & $-1.277245285008(+1)$ \\
\hline 27 & $-1.007317532629(+2)$ & 73 & $-3.173751593817(+1)$ & 119 & $-1.246653869129(+1)$ \\
\hline 28 & $-9.696575588466(+1)$ & 74 & $-3.113053020237(+1)$ & 120 & $-1.216337230938(+1)$ \\
\hline 29 & $-9.345348534052(+1)$ & 75 & $-3.053706453634(+1)$ & 121 & $-1.186285919145(+1)$ \\
\hline 30 & $-9.016956009213(+1)$ & 76 & $-2.995657227701(+1)$ & 122 & $-1.156490715746(+1)$ \\
\hline 31 & $-8.709187330046(+1)$ & 77 & $-2.938853483843(+1)$ & 123 & $-1.126942623829(+1)$ \\
\hline 32 & $-8.420108115013(+1)$ & 78 & $-2.883245990403(+1)$ & 124 & $-1.097632855862(+1)$ \\
\hline 33 & $-8.148018420549(+1)$ & 79 & $-2.828787975591(+1)$ & 125 & $-1.068552822412(+1)$ \\
\hline 34 & $-7.891418264493(+1)$ & 80 & $-2.775434972921(+1)$ & 126 & $-1.039694121284(+1)$ \\
\hline 35 & $-7.648979059765(+1)$ & 81 & $-2.723144678065(+1)$ & 127 & $-1.011048527031(+1)$ \\
\hline 36 & $-7.419519809088(+1)$ & 82 & $-2.671876816148(+1)$ & 128 & $-9.826079808224(+0)$ \\
\hline 37 & $-7.201987160001(+1)$ & 83 & $-2.621593018616(+1)$ & 129 & $-9.543645806388(+0)$ \\
\hline 38 & $-6.995438609077(+1)$ & 84 & $-2.572256708861(+1)$ & 130 & $-9.263105717640(+0)$ \\
\hline 39 & $-6.799028290080(+1)$ & 85 & $-2.523832995892(+1)$ & 131 & $-8.984383375590(+0)$ \\
\hline 40 & $-6.611994893890(+1)$ & 86 & $-2.476288575390(+1)$ & 132 & $-8.707403904875(+0)$ \\
\hline 41 & $-6.433651356224(+1)$ & 87 & $-2.429591637556(+1)$ & 133 & $-8.432093633740(+0)$ \\
\hline 42 & $-6.263376018530(+1)$ & 88 & $-2.383711781208(+1)$ & 134 & $-8.158380008720(+0)$ \\
\hline 43 & $-6.100605022221(+1)$ & 89 & $-2.338619933633(+1)$ & 135 & $-7.886191511223(+0)$ \\
\hline 44 & $-5.944825740051(+1)$ & 90 & $-2.294288275746(+1)$ & 136 & $-7.615457575802(+0)$ \\
\hline 45 & $-5.795571083286(+1)$ & 91 & $-2.250690172 \quad 150(+1)$ & 137 & $-7.346108509931(+0)$ \\
\hline 46 & $-5.652414551403(+1)$ & 92 & $-2.207800105715(+1)$ & & \\
\hline
\end{tabular}


Table 5

Magnetic shielding constants $\sigma$ (in units of $\alpha^{2}$ ) of Dirac one-electron atoms in the excited state $2 \mathrm{p}_{3 / 2}(\mu= \pm 3 / 2)$. The number in brackets is the power of 10 by which the corresponding entry is to be multiplied. The value of the inverse of the fine-structure constant used was $\alpha^{-1}=137.035999139$ (from CODATA 2014).

\begin{tabular}{|c|c|c|c|c|c|}
\hline$Z$ & $\sigma\left[\alpha^{2}\right]$ & $Z$ & $\sigma\left[\alpha^{2}\right]$ & $Z$ & $\sigma\left[\alpha^{2}\right]$ \\
\hline 1 & $1.000028534293(-1)$ & 47 & $5.006732203585(+0)$ & 93 & $1.194851329368(+1)$ \\
\hline 2 & $2.000228284951(-1)$ & 48 & $5.127227928174(+0)$ & 94 & $1.214396435565(+1)$ \\
\hline 3 & $3.000770521391(-1)$ & 49 & $5.248650771844(+0)$ & 95 & $1.234202117158(+1)$ \\
\hline 4 & $4.001826619163(-1)$ & 50 & $5.371024936168(+0)$ & 96 & $1.254274039325(+1)$ \\
\hline 5 & $5.003568113104(-1)$ & 51 & $5.494374957625(+0)$ & 97 & $1.274617999639(+1)$ \\
\hline 6 & $6.006166750591(-1)$ & 52 & $5.618725718030(+0)$ & 98 & $1.295239932296(+1)$ \\
\hline 7 & $7.009794544924(-1)$ & 53 & $5.744102455240(+0)$ & 99 & $1.316145912505(+1)$ \\
\hline 8 & $8.014623828881(-1)$ & 54 & $5.870530774109(+0)$ & 100 & $1.337342161028(+1)$ \\
\hline 9 & $9.020827308481(-1)$ & 55 & $5.998036657744(+0)$ & 101 & $1.358835048900(+1)$ \\
\hline 10 & $1.002857811697(+0)$ & 56 & $6.126646479032(+0)$ & 102 & $1.380631102320(+1)$ \\
\hline 11 & $1.103804986909(+0)$ & 57 & $6.256387012479(+0)$ & 103 & $1.402737007727(+1)$ \\
\hline 12 & $1.204941671566(+0)$ & 58 & $6.387285446364(+0)$ & 104 & $1.425159617068(+1)$ \\
\hline 13 & $1.306285339846(+0)$ & 59 & $6.519369395201(+0)$ & 105 & $1.447905953270(+1)$ \\
\hline 14 & $1.407853530555(+0)$ & 60 & $6.652666912562(+0)$ & 106 & $1.470983215919(+1)$ \\
\hline 15 & $1.509663852696(+0)$ & 61 & $6.787206504222(+0)$ & 107 & $1.494398787160(+1)$ \\
\hline 16 & $1.611733991085(+0)$ & 62 & $6.923017141690(+0)$ & 108 & $1.518160237830(+1)$ \\
\hline 17 & $1.714081712011(+0)$ & 63 & $7.060128276102(+0)$ & 109 & $1.542275333825(+1)$ \\
\hline 18 & $1.816724868958(+0)$ & 64 & $7.198569852506(+0)$ & 110 & $1.566752042724(+1)$ \\
\hline 19 & $1.919681408371(+0)$ & 65 & $7.338372324559(+0)$ & 111 & $1.591598540679(+1)$ \\
\hline 20 & $2.022969375495(+0)$ & 66 & $7.479566669634(+0)$ & 112 & $1.616823219571(+1)$ \\
\hline 21 & $2.126606920262(+0)$ & 67 & $7.622184404374(+0)$ & 113 & $1.642434694463(+1)$ \\
\hline 22 & $2.230612303263(+0)$ & 68 & $7.766257600692(+0)$ & 114 & $1.668441811354(+1)$ \\
\hline 23 & $2.335003901781(+0)$ & 69 & $7.911818902242(+0)$ & 115 & $1.694853655251(+1)$ \\
\hline 24 & $2.439800215902(+0)$ & 70 & $8.058901541383(+0)$ & 116 & $1.721679558568(+1)$ \\
\hline 25 & $2.545019874708(+0)$ & 71 & $8.207539356645(+0)$ & 117 & $1.748929109879(+1)$ \\
\hline 26 & $2.650681642553(+0)$ & 72 & $8.357766810726(+0)$ & 118 & $1.776612163038(+1)$ \\
\hline 27 & $2.756804425433(+0)$ & 73 & $8.509619009031(+0)$ & 119 & $1.804738846673(+1)$ \\
\hline 28 & $2.863407277441(+0)$ & 74 & $8.663131718786(+0)$ & 120 & $1.833319574096(+1)$ \\
\hline 29 & $2.970509407327(+0)$ & 75 & $8.818341388736(+0)$ & 121 & $1.862365053620(+1)$ \\
\hline 30 & $3.078130185159(+0)$ & 76 & $8.975285169462(+0)$ & 122 & $1.891886299335(+1)$ \\
\hline 31 & $3.186289149091(+0)$ & 77 & $9.134000934331(+0)$ & 123 & $1.921894642340(+1)$ \\
\hline 32 & $3.295006012245(+0)$ & 78 & $9.294527301111(+0)$ & 124 & $1.952401742466(+1)$ \\
\hline 33 & $3.404300669711(+0)$ & 79 & $9.456903654276(+0)$ & 125 & $1.983419600518(+1)$ \\
\hline 34 & $3.514193205667(+0)$ & 80 & $9.621170168023(+0)$ & 126 & $2.014960571055(+1)$ \\
\hline 35 & $3.624703900634(+0)$ & 81 & $9.787367830040(+0)$ & 127 & $2.047037375737(+1)$ \\
\hline 36 & $3.735853238857(+0)$ & 82 & $9.955538466039(+0)$ & 128 & $2.079663117281(+1)$ \\
\hline 37 & $3.847661915832(+0)$ & 83 & $1.012572476510(+1)$ & 129 & $2.112851294034(+1)$ \\
\hline 38 & $3.960150845976(+0)$ & 84 & $1.029797030587(+1)$ & 130 & $2.146615815223(+1)$ \\
\hline 39 & $4.073341170449(+0)$ & 85 & $1.047231958359(+1)$ & 131 & $2.180971016896(+1)$ \\
\hline 40 & $4.187254265134(+0)$ & 86 & $1.064881803806(+1)$ & 132 & $2.215931678605(+1)$ \\
\hline 41 & $4.301911748780(+0)$ & 87 & $1.082751208257(+1)$ & 133 & $2.251513040866(+1)$ \\
\hline 42 & $4.417335491320(+0)$ & 88 & $1.100844913376(+1)$ & 134 & $2.287730823434(+1)$ \\
\hline 43 & $4.533547622359(+0)$ & 89 & $1.119167764254(+1)$ & 135 & $2.324601244454(+1)$ \\
\hline 44 & $4.650570539855(+0)$ & 90 & $1.137724712608(+1)$ & 136 & $2.362141040512(+1)$ \\
\hline 45 & $4.768426918985(+0)$ & 91 & $1.156520820089(+1)$ & 137 & $2.400367487661(+1)$ \\
\hline 46 & $4.887139721218(+0)$ & 92 & $1.175561261705(+1)$ & & \\
\hline
\end{tabular}


Table 6

Magnetic shielding constants $\sigma$ (in units of $\alpha^{2}$ ) of Dirac one-electron atoms in the excited state $3 \mathrm{~s}_{1 / 2}$. The number in brackets is the power of 10 by which the corresponding entry is to be multiplied. The value of the inverse of the fine-structure constant used was $\alpha^{-1}=137.035999139$ (from CODATA 2014).

\begin{tabular}{|c|c|c|c|c|c|}
\hline$Z$ & $\sigma\left[\alpha^{2}\right]$ & $Z$ & $\sigma\left[\alpha^{2}\right]$ & $Z$ & $\sigma\left[\alpha^{2}\right]$ \\
\hline 1 & $3.703923474808(-2)$ & 41 & $1.698307552238(+0)$ & 81 & $5.725125585478(+0)$ \\
\hline 2 & $7.409166080451(-2)$ & 42 & $1.750586151557(+0)$ & 82 & $5.948718328129(+0)$ \\
\hline 3 & $1.111704947074(-1)$ & 43 & $1.803857232510(+0)$ & 83 & $6.186797501693(+0)$ \\
\hline 4 & $1.482890035448(-1)$ & 44 & $1.858174655865(+0)$ & 84 & $6.440797647070(+0)$ \\
\hline 5 & $1.854605304549(-1)$ & 45 & $1.913595512887(+0)$ & 85 & $6.712336790020(+0)$ \\
\hline 6 & $2.226985204001(-1)$ & 46 & $1.970180375848(+0)$ & 86 & $7.003245748591(+0)$ \\
\hline 7 & $2.600165463054(-1)$ & 47 & $2.027993570833(+0)$ & 87 & $7.315603132682(+0)$ \\
\hline 8 & $2.974283356588(-1)$ & 48 & $2.087103475176(+0)$ & 88 & $7.651777353963(+0)$ \\
\hline 9 & $3.349477976707(-1)$ & 49 & $2.147582842152(+0)$ & 89 & $8.014477322932(+0)$ \\
\hline 10 & $3.725890510936(-1)$ & 50 & $2.209509155821(+0)$ & 90 & $8.406813981412(+0)$ \\
\hline 11 & $4.103664528074(-1)$ & 51 & $2.272965019336(+0)$ & 91 & $8.832375444528(+0)$ \\
\hline 12 & $4.482946272799(-1)$ & 52 & $2.338038580388(+0)$ & 92 & $9.295319363853(+0)$ \\
\hline 13 & $4.863884970172(-1)$ & 53 & $2.404823997949(+0)$ & 93 & $9.800487255876(+0)$ \\
\hline 14 & $5.246633141248(-1)$ & 54 & $2.473421955003(+0)$ & 94 & $1.035354708691(+1)$ \\
\hline 15 & $5.631346931066(-1)$ & 55 & $2.543940222552(+0)$ & 95 & $1.096117254246(+1)$ \\
\hline 16 & $6.018186450373(-1)$ & 56 & $2.616494280894(+0)$ & 96 & $1.163127039630(+1)$ \\
\hline 17 & $6.407316132504(-1)$ & 57 & $2.691208004969(+0)$ & 97 & $1.237327162567(+1)$ \\
\hline 18 & $6.798905106957(-1)$ & 58 & $2.768214421486(+0)$ & 98 & $1.319850799508(+1)$ \\
\hline 19 & $7.193127591287(-1)$ & 59 & $2.847656546617(+0)$ & 99 & $1.412070469260(+1)$ \\
\hline 20 & $7.590163303074(-1)$ & 60 & $2.929688314272(+0)$ & 100 & $1.515663274100(+1)$ \\
\hline 21 & $7.990197893839(-1)$ & 61 & $3.014475606402(+0)$ & 101 & $1.632698474256(+1)$ \\
\hline 22 & $8.393423406928(-1)$ & 62 & $3.102197398437(+0)$ & 102 & $1.765756807248(+1)$ \\
\hline 23 & $8.800038761551(-1)$ & 63 & $3.193047034904(+0)$ & 103 & $1.918095774939(+1)$ \\
\hline 24 & $9.210250265322(-1)$ & 64 & $3.287233652513(+0)$ & 104 & $2.093882885492(+1)$ \\
\hline 25 & $9.624272157863(-1)$ & 65 & $3.384983770672(+0)$ & 105 & $2.298531714264(+1)$ \\
\hline 26 & $1.004232718823(+0)$ & 66 & $3.486543072483(+0)$ & 106 & $2.539197671391(+1)$ \\
\hline 27 & $1.046464722914(+0)$ & 67 & $3.592178402917(+0)$ & 107 & $2.825529369226(+1)$ \\
\hline 28 & $1.089147393139(+0)$ & 68 & $3.702180015200(+0)$ & 108 & $3.170843377905(+1)$ \\
\hline 29 & $1.132305942176(+0)$ & 69 & $3.816864101559(+0)$ & 109 & $3.594028934195(+1)$ \\
\hline 30 & $1.175966704866(+0)$ & 70 & $3.936575650516(+0)$ & 110 & $4.122771915489(+1)$ \\
\hline 31 & $1.220157217942(+0)$ & 71 & $4.061691680195(+0)$ & 111 & $4.799301658298(+1)$ \\
\hline 32 & $1.264906305410(+0)$ & 72 & $4.192624905734(+0)$ & 112 & $5.691306540264(+1)$ \\
\hline 33 & $1.310244170078(+0)$ & 73 & $4.329827909268(+0)$ & 113 & $6.914393412568(+1)$ \\
\hline 34 & $1.356202491797(+0)$ & 74 & $4.473797893488(+0)$ & 114 & $8.683371886153(+1)$ \\
\hline 35 & $1.402814533026(+0)$ & 75 & $4.625082114826(+0)$ & 115 & $1.144724698887(+2)$ \\
\hline 36 & $1.450115252398(+0)$ & 76 & $4.784284110729(+0)$ & 116 & $1.632684158293(+2)$ \\
\hline 37 & $1.498141427021(+0)$ & 77 & $4.952070857746(+0)$ & 117 & $2.711853575923(+2)$ \\
\hline 38 & $1.546931784343(+0)$ & 78 & $5.129181024556(+0)$ & 118 & $7.006562986455(+2)$ \\
\hline 39 & $1.596527144476(+0)$ & 79 & $5.316434517645(+0)$ & & \\
\hline 40 & $1.646970573984(+0)$ & 80 & $5.514743558845(+0)$ & & \\
\hline
\end{tabular}


Table 7

Magnetic shielding constants $\sigma$ (in units of $\alpha^{2}$ ) of Dirac one-electron atoms in the excited state $3 \mathrm{p}_{1 / 2}$. The number in brackets is the power of 10 by which the corresponding entry is to be multiplied. The value of the inverse of the fine-structure constant used was $\alpha^{-1}=137.035999139$ (from CODATA 2014).

\begin{tabular}{|c|c|c|c|c|c|}
\hline$Z$ & $\sigma\left[\alpha^{2}\right]$ & $Z$ & $\sigma\left[\alpha^{2}\right]$ & $Z$ & $\sigma\left[\alpha^{2}\right]$ \\
\hline 1 & $2.782082890470(+3)$ & 41 & $6.910908149276(+1)$ & 81 & $3.768455123575(+1)$ \\
\hline 2 & $1.391084662008(+3)$ & 42 & $6.752836629589(+1)$ & 82 & $3.735948908009(+1)$ \\
\hline 3 & $9.274378055773(+2)$ & 43 & $6.602317513960(+1)$ & 83 & $3.704927797438(+1)$ \\
\hline 4 & $6.956288059806(+2)$ & 44 & $6.458840370844(+1)$ & 84 & $3.675401178243(+1)$ \\
\hline 5 & $5.565549646388(+2)$ & 45 & $6.321940416759(+1)$ & 85 & $3.647385858656(+1)$ \\
\hline 6 & $4.638487191071(+2)$ & 46 & $6.191193574145(+1)$ & 86 & $3.620906878910(+1)$ \\
\hline 7 & $3.976382601559(+2)$ & 47 & $6.066212161873(+1)$ & 87 & $3.595998504140(+1)$ \\
\hline 8 & $3.479876860479(+2)$ & 48 & $5.946641126318(+1)$ & 88 & $3.572705440162(+1)$ \\
\hline 9 & $3.093770539701(+2)$ & 49 & $5.832154735956(+1)$ & 89 & $3.551084323375(+1)$ \\
\hline 10 & $2.784944006127(+2)$ & 50 & $5.722453674811(+1)$ & 90 & $3.531205550596(+1)$ \\
\hline 11 & $2.532321151645(+2)$ & 51 & $5.617262480245(+1)$ & 91 & $3.513155533989(+1)$ \\
\hline 12 & $2.321851258161(+2)$ & 52 & $5.516327278985(+1)$ & 92 & $3.497039492050(+1)$ \\
\hline 13 & $2.143806924971(+2)$ & 53 & $5.419413782287(+1)$ & 93 & $3.482984922609(+1)$ \\
\hline 14 & $1.991240027311(+2)$ & 54 & $5.326305506963(+1)$ & 94 & $3.471145951425(+1)$ \\
\hline 15 & $1.859055291532(+2)$ & 55 & $5.236802193870(+1)$ & 95 & $3.461708815888(+1)$ \\
\hline 16 & $1.743431279627(+2)$ & 56 & $5.150718399566(+1)$ & 96 & $3.454898835331(+1)$ \\
\hline 17 & $1.641445732216(+2)$ & 57 & $5.067882240303(+1)$ & 97 & $3.450989349864(+1)$ \\
\hline 18 & $1.550825797236(+2)$ & 58 & $4.988134270460(+1)$ & 98 & $3.450313296775(+1)$ \\
\hline 19 & $1.469777133836(+2)$ & 59 & $4.911326479992(+1)$ & 99 & $3.453278366550(+1)$ \\
\hline 20 & $1.396864285149(+2)$ & 60 & $4.837321397631(+1)$ & 100 & $3.460387085178(+1)$ \\
\hline 21 & $1.330925230312(+2)$ & 61 & $4.765991288379(+1)$ & 101 & $3.472263780408(+1)$ \\
\hline 22 & $1.271009240538(+2)$ & 62 & $4.697217435415(+1)$ & 102 & $3.489691330579(+1)$ \\
\hline 23 & $1.216330946687(+2)$ & 63 & $4.630889497914(+1)$ & 103 & $3.513662076432(+1)$ \\
\hline 24 & $1.166235889998(+2)$ & 64 & $4.566904937439(+1)$ & 104 & $3.545449667254(+1)$ \\
\hline 25 & $1.120174340699(+2)$ & 65 & $4.505168506668(+1)$ & 105 & $3.586712578046(+1)$ \\
\hline 26 & $1.077681158524(+2)$ & 66 & $4.445591795079(+1)$ & 106 & $3.639646816218(+1)$ \\
\hline 27 & $1.038360128725(+2)$ & 67 & $4.388092827129(+1)$ & 107 & $3.707217347249(+1)$ \\
\hline 28 & $1.001871654717(+2)$ & 68 & $4.332595709156(+1)$ & 108 & $3.793519906978(+1)$ \\
\hline 29 & $9.679229971192(+1)$ & 69 & $4.279030321984(+1)$ & 109 & $3.904367600688(+1)$ \\
\hline 30 & $9.362604650627(+1)$ & 70 & $4.227332056852(+1)$ & 110 & $4.048283752422(+1)$ \\
\hline 31 & $9.066631189104(+1)$ & 71 & $4.177441592928(+1)$ & 111 & $4.238271612142(+1)$ \\
\hline 32 & $8.789376537916(+1)$ & 72 & $4.129304715310(+1)$ & 112 & $4.495175649644(+1)$ \\
\hline 33 & $8.529142134710(+1)$ & 73 & $4.082872173044(+1)$ & 113 & $4.854597436902(+1)$ \\
\hline 34 & $8.284429430218(+1)$ & 74 & $4.038099577366(+1)$ & 114 & $5.382687229570(+1)$ \\
\hline 35 & $8.053911325475(+1)$ & 75 & $3.994947341072(+1)$ & 115 & $6.217710776460(+1)$ \\
\hline 36 & $7.836408370333(+1)$ & 76 & $3.953380660727(+1)$ & 116 & $7.704800767952(+1)$ \\
\hline 37 & $7.630868822552(+1)$ & 77 & $3.913369544286(+1)$ & 117 & $1.101294081066(+2)$ \\
\hline 38 & $7.436351856396(+1)$ & 78 & $3.874888887715(+1)$ & 118 & $2.422288410147(+2)$ \\
\hline 39 & $7.252013355511(+1)$ & 79 & $3.837918605407(+1)$ & & \\
\hline 40 & $7.077093837929(+1)$ & 80 & $3.802443820582(+1)$ & & \\
\hline
\end{tabular}


Table 8

Magnetic shielding constants $\sigma$ (in units of $\alpha^{2}$ ) of Dirac one-electron atoms in the excited state $3 \mathrm{p}_{3 / 2}(\mu= \pm 1 / 2)$. The number in brackets is the power of 10 by which the corresponding entry is to be multiplied. The value of the inverse of the fine-structure constant used was $\alpha^{-1}=137.035999139$ (from CODATA 2014).

\begin{tabular}{|c|c|c|c|c|c|}
\hline$Z$ & $\sigma\left[\alpha^{2}\right]$ & $Z$ & $\sigma\left[\alpha^{2}\right]$ & $Z$ & $\sigma\left[\alpha^{2}\right]$ \\
\hline 1 & $-2.782016222669(+3)$ & 47 & $-5.739014103697(+1)$ & 93 & $-2.619661547266(+1)$ \\
\hline 2 & $-1.390951319594(+3)$ & 48 & $-5.611756338335(+1)$ & 94 & $-2.583348334573(+1)$ \\
\hline 3 & $-9.272377749217(+2)$ & 49 & $-5.489525051725(+1)$ & 95 & $-2.547686251 \quad 175(+1)$ \\
\hline 4 & $-6.953620666150(+2)$ & 50 & $-5.372018064047(+1)$ & 96 & $-2.512653963211(+1)$ \\
\hline 5 & $-5.562214892304(+2)$ & 51 & $-5.258956879768(+1)$ & 97 & $-2.478230991076(+1)$ \\
\hline 6 & $-4.634484734216(+2)$ & 52 & $-5.150084410021(+1)$ & 98 & $-2.444397665079(+1)$ \\
\hline 7 & $-3.971712030117(+2)$ & 53 & $-5.045162952819(+1)$ & 99 & $-2.411135083748(+1)$ \\
\hline 8 & $-3.474537692607(+2)$ & 54 & $-4.943972397676(+1)$ & 100 & $-2.378425074608(+1)$ \\
\hline 9 & $-3.087762222882(+2)$ & 55 & $-4.846308626081(+1)$ & 101 & $-2.346250157263(+1)$ \\
\hline 10 & $-2.778265916417(+2)$ & 56 & $-4.751982083340(+1)$ & 102 & $-2.314593508609(+1)$ \\
\hline 11 & $-2.524972592825(+2)$ & 57 & $-4.660816500732(+1)$ & 103 & $-2.283438930047(+1)$ \\
\hline 12 & $-2.313831460779(+2)$ & 58 & $-4.572647749860(+1)$ & 104 & $-2.252770816546(+1)$ \\
\hline 13 & $-2.135115045286(+2)$ & 59 & $-4.487322813483(+1)$ & 105 & $-2.222574127442(+1)$ \\
\hline 14 & $-1.981875146112(+2)$ & 60 & $-4.404698859264(+1)$ & 106 & $-2.192834358849(+1)$ \\
\hline 15 & $-1.849016412854(+2)$ & 61 & $-4.324642404609(+1)$ & 107 & $-2.163537517581(+1)$ \\
\hline 16 & $-1.732717329342(+2)$ & 62 & $-4.247028562317(+1)$ & 108 & $-2.134670096479(+1)$ \\
\hline 17 & $-1.630055556498(+2)$ & 63 & $-4.171740358065(+1)$ & 109 & $-2.106219051055(+1)$ \\
\hline 18 & $-1.538758160899(+2)$ & 64 & $-4.098668111863(+1)$ & 110 & $-2.078171777368(+1)$ \\
\hline 19 & $-1.457030718536(+2)$ & 65 & $-4.027708876595(+1)$ & 111 & $-2.050516091047(+1)$ \\
\hline 20 & $-1.383437687439(+2)$ & 66 & $-3.958765927589(+1)$ & 112 & $-2.023240207397(+1)$ \\
\hline 21 & $-1.316816959554(+2)$ & 67 & $-3.891748297888(+1)$ & 113 & $-1.996332722501(+1)$ \\
\hline 22 & $-1.256217716650(+2)$ & 68 & $-3.826570354511(+1)$ & 114 & $-1.969782595276(+1)$ \\
\hline 23 & $-1.200854497732(+2)$ & 69 & $-3.763151411544(+1)$ & 115 & $-1.943579130410(+1)$ \\
\hline 24 & $-1.150072749592(+2)$ & 70 & $-3.701415376394(+1)$ & 116 & $-1.917711962119(+1)$ \\
\hline 25 & $-1.103322645236(+2)$ & 71 & $-3.641290425908(+1)$ & 117 & $-1.892171038690(+1)$ \\
\hline 26 & $-1.060138944201(+2)$ & 72 & $-3.582708709478(+1)$ & 118 & $-1.866946607742(+1)$ \\
\hline 27 & $-1.020125328369(+2)$ & 73 & $-3.525606076537(+1)$ & 119 & $-1.842029202171(+1)$ \\
\hline 28 & $-9.829420943764(+1)$ & 74 & $-3.469921826121(+1)$ & 120 & $-1.817409626731(+1)$ \\
\hline 29 & $-9.482963924340(+1)$ & 75 & $-3.415598476471(+1)$ & 121 & $-1.793078945215(+1)$ \\
\hline 30 & $-9.159344173736(+1)$ & 76 & $-3.362581552792(+1)$ & 122 & $-1.769028468193(+1)$ \\
\hline 31 & $-8.856351111072(+1)$ & 77 & $-3.310819391544(+1)$ & 123 & $-1.745249741272(+1)$ \\
\hline 32 & $-8.572050458736(+1)$ & 78 & $-3.260262959771(+1)$ & 124 & $-1.721734533856(+1)$ \\
\hline 33 & $-8.304742378030(+1)$ & 79 & $-3.210865688131(+1)$ & 125 & $-1.698474828351(+1)$ \\
\hline 34 & $-8.052926992630(+1)$ & 80 & $-3.162583316438(+1)$ & 126 & $-1.675462809811(+1)$ \\
\hline 35 & $-7.815275822307(+1)$ & 81 & $-3.115373750624(+1)$ & 127 & $-1.652690855982(+1)$ \\
\hline 36 & $-7.590607977682(+1)$ & 82 & $-3.069196930148(+1)$ & 128 & $-1.630151527713(+1)$ \\
\hline 37 & $-7.377870215282(+1)$ & 83 & $-3.024014704980(+1)$ & 129 & $-1.607837559731(+1)$ \\
\hline 38 & $-7.176120141789(+1)$ & 84 & $-2.979790721344(+1)$ & 130 & $-1.585741851724(+1)$ \\
\hline 39 & $-6.984512002240(+1)$ & 85 & $-2.936490315510(+1)$ & 131 & $-1.563857459739(+1)$ \\
\hline 40 & $-6.802284599995(+1)$ & 86 & $-2.894080414980(+1)$ & 132 & $-1.542177587854(+1)$ \\
\hline 41 & $-6.628750984496(+1)$ & 87 & $-2.852529446464(+1)$ & 133 & $-1.520695580111(+1)$ \\
\hline 42 & $-6.463289612200(+1)$ & 88 & $-2.811807250107(+1)$ & 134 & $-1.499404912687(+1)$ \\
\hline 43 & $-6.305336740860(+1)$ & 89 & $-2.771884999488(+1)$ & 135 & $-1.478299186285(+1)$ \\
\hline 44 & $-6.154379860940(+1)$ & 90 & $-2.732735126918(+1)$ & 136 & $-1.457372118729(+1)$ \\
\hline 45 & $-6.009952002835(+1)$ & 91 & $-2.694331253645(+1)$ & 137 & $-1.436617537737(+1)$ \\
\hline 46 & $-5.871626786613(+1)$ & 92 & $-2.656648124590(+1)$ & & \\
\hline
\end{tabular}


Table 9

Magnetic shielding constants $\sigma$ (in units of $\alpha^{2}$ ) of Dirac one-electron atoms in the excited state $3 \mathrm{p}_{3 / 2}(\mu= \pm 3 / 2)$. The number in brackets is the power of 10 by which the corresponding entry is to be multiplied. The value of the inverse of the fine-structure constant used was $\alpha^{-1}=137.035999139$ (from CODATA 2014).

\begin{tabular}{|c|c|c|c|c|c|}
\hline$Z$ & $\sigma\left[\alpha^{2}\right]$ & $Z$ & $\sigma\left[\alpha^{2}\right]$ & $Z$ & $\sigma\left[\alpha^{2}\right]$ \\
\hline 1 & $4.444542008200(-2)$ & 47 & $2.194987914398(+0)$ & 93 & $5.083238724003(+0)$ \\
\hline 2 & $8.889669447279(-2)$ & 48 & $2.246579299652(+0)$ & 94 & $5.163010060919(+0)$ \\
\hline 3 & $1.333596798990(-1)$ & 49 & $2.298500313290(+0)$ & 95 & $5.243811083108(+0)$ \\
\hline 4 & $1.778402379248(-1)$ & 50 & $2.350760108989(+0)$ & 96 & $5.325667295 \quad 712(+0)$ \\
\hline 5 & $2.223442373750(-1)$ & 51 & $2.403367999735(+0)$ & 97 & $5.408604900878(+0)$ \\
\hline 6 & $2.668775567636(-1)$ & 52 & $2.456333463074(+0)$ & 98 & $5.492650821928(+0)$ \\
\hline 7 & $3.114460867302(-1)$ & 53 & $2.509666146488(+0)$ & 99 & $5.577832728494(+0)$ \\
\hline 8 & $3.560557324843(-1)$ & 54 & $2.563375872945(+0)$ & 100 & $5.664179062647(+0)$ \\
\hline 9 & $4.007124162612(-1)$ & 55 & $2.617472646587(+0)$ & 101 & $5.751719066073(+0)$ \\
\hline 10 & $4.454220797908(-1)$ & 56 & $2.671966658592(+0)$ & 102 & $5.840482808346(+0)$ \\
\hline 11 & $4.901906867805(-1)$ & 57 & $2.726868293206(+0)$ & 103 & $5.930501216344(+0)$ \\
\hline 12 & $5.350242254146(-1)$ & 58 & $2.782188133950(+0)$ & 104 & $6.021806104865(+0)$ \\
\hline 13 & $5.799287108724(-1)$ & 59 & $2.837936970007(+0)$ & 105 & $6.114430208506(+0)$ \\
\hline 14 & $6.249101878663(-1)$ & 60 & $2.894125802809(+0)$ & 106 & $6.208407214850(+0)$ \\
\hline 15 & $6.699747332021(-1)$ & 61 & $2.950765852815(+0)$ & 107 & $6.303771799040(+0)$ \\
\hline 16 & $7.151284583638(-1)$ & 62 & $3.007868566502(+0)$ & 108 & $6.400559659794(+0)$ \\
\hline 17 & $7.603775121240(-1)$ & 63 & $3.065445623562(+0)$ & 109 & $6.498807556946(+0)$ \\
\hline 18 & $8.057280831832(-1)$ & 64 & $3.123508944337(+0)$ & 110 & $6.598553350564(+0)$ \\
\hline 19 & $8.511864028389(-1)$ & 65 & $3.182070697466(+0)$ & 111 & $6.699836041752(+0)$ \\
\hline 20 & $8.967587476874(-1)$ & 66 & $3.241143307798(+0)$ & 112 & $6.802695815194(+0)$ \\
\hline 21 & $9.424514423596(-1)$ & 67 & $3.300739464531(+0)$ & 113 & $6.907174083538(+0)$ \\
\hline 22 & $9.882708622943(-1)$ & 68 & $3.360872129636(+0)$ & 114 & $7.013313533718(+0)$ \\
\hline 23 & $1.034223436550(+0)$ & 69 & $3.421554546533(+0)$ & 115 & $7.121158175290(+0)$ \\
\hline 24 & $1.080315650657(+0)$ & 70 & $3.482800249064(+0)$ & 116 & $7.230753390916(+0)$ \\
\hline 25 & $1.126554049517(+0)$ & 71 & $3.544623070756(+0)$ & 117 & $7.342145989073(+0)$ \\
\hline 26 & $1.172945240340(+0)$ & 72 & $3.607037154384(+0)$ & 118 & $7.455384259126(+0)$ \\
\hline 27 & $1.219495895642(+0)$ & 73 & $3.670056961863(+0)$ & 119 & $7.570518028 \quad 870(+0)$ \\
\hline 28 & $1.266212756280(+0)$ & 74 & $3.733697284465(+0)$ & 120 & $7.687598724686(+0)$ \\
\hline 29 & $1.313102634554(+0)$ & 75 & $3.797973253386(+0)$ & 121 & $7.806679434429(+0)$ \\
\hline 30 & $1.360172417352(+0)$ & 76 & $3.862900350665(+0)$ & 122 & $7.927814973205(+0)$ \\
\hline 31 & $1.407429069364(+0)$ & 77 & $3.928494420490(+0)$ & 123 & $8.051061952193(+0)$ \\
\hline 32 & $1.454879636352(+0)$ & 78 & $3.994771680880(+0)$ & 124 & $8.176478850660(+0)$ \\
\hline 33 & $1.502531248486(+0)$ & 79 & $4.061748735782(+0)$ & 125 & $8.304126091351(+0)$ \\
\hline 34 & $1.550391123748(+0)$ & 80 & $4.129442587592(+0)$ & 126 & $8.434066119441(+0)$ \\
\hline 35 & $1.598466571404(+0)$ & 81 & $4.197870650111(+0)$ & 127 & $8.566363485232(+0)$ \\
\hline 36 & $1.646764995554(+0)$ & 82 & $4.267050761964(+0)$ & 128 & $8.701084930806(+0)$ \\
\hline 37 & $1.695293898752(+0)$ & 83 & $4.337001200500(+0)$ & 129 & $8.838299480 \quad 853(+0)$ \\
\hline 38 & $1.744060885713(+0)$ & 84 & $4.407740696188(+0)$ & 130 & $8.978078537913(+0)$ \\
\hline 39 & $1.793073667098(+0)$ & 85 & $4.479288447534(+0)$ & 131 & $9.120495982258(+0)$ \\
\hline 40 & $1.842340063390(+0)$ & 86 & $4.551664136542(+0)$ & 132 & $9.265628276 \quad 706(+0)$ \\
\hline 41 & $1.891868008857(+0)$ & 87 & $4.624887944747(+0)$ & 133 & $9.413554576624(+0)$ \\
\hline 42 & $1.941665555613(+0)$ & 88 & $4.698980569824(+0)$ & 134 & $9.564356845429(+0)$ \\
\hline 43 & $1.991740877772(+0)$ & 89 & $4.773963242834(+0)$ & 135 & $9.718119975899(+0)$ \\
\hline 44 & $2.042102275713(+0)$ & 90 & $4.849857746095(+0)$ & 136 & $9.874931917637(+0)$ \\
\hline 45 & $2.092758180441(+0)$ & 91 & $4.926686431739(+0)$ & 137 & $1.003488381104(+1)$ \\
\hline 46 & $2.143717158067(+0)$ & 92 & $5.004472240959(+0)$ & & \\
\hline
\end{tabular}


Table 10

Magnetic shielding constants $\sigma$ (in units of $\alpha^{2}$ ) of Dirac one-electron atoms in the excited state $3 \mathrm{~d}_{3 / 2}(\mu= \pm 1 / 2)$. The number in brackets is the power of 10 by which the corresponding entry is to be multiplied. The value of the inverse of the fine-structure constant used was $\alpha^{-1}=137.035999139$ (from CODATA 2014).

\begin{tabular}{|c|c|c|c|c|c|}
\hline$Z$ & $\sigma\left[\alpha^{2}\right]$ & $Z$ & $\sigma\left[\alpha^{2}\right]$ & $Z$ & $\sigma\left[\alpha^{2}\right]$ \\
\hline 1 & $1.802795342177(+3)$ & 47 & $3.951137250530(+1)$ & 93 & $2.175154669680(+1)$ \\
\hline 2 & $9.014341164174(+2)$ & 48 & $3.873740825098(+1)$ & 94 & $2.157344556779(+1)$ \\
\hline 3 & $6.009965739946(+2)$ & 49 & $3.799609803823(+1)$ & 95 & $2.139978768223(+1)$ \\
\hline 4 & $4.507899541760(+2)$ & 50 & $3.728548584369(+1)$ & 96 & $2.123043947913(+1)$ \\
\hline 5 & $3.606757053822(+2)$ & 51 & $3.660376914457(+1)$ & 97 & $2.106527303938(+1)$ \\
\hline 6 & $3.006076442268(+2)$ & 52 & $3.594928416047(+1)$ & 98 & $2.090416580185(+1)$ \\
\hline 7 & $2.577088353792(+2)$ & 53 & $3.532049276600(+1)$ & 99 & $2.074700029684(+1)$ \\
\hline 8 & $2.255408115306(+2)$ & 54 & $3.471597085764(+1)$ & 100 & $2.059366389564(+1)$ \\
\hline 9 & $2.005266467157(+2)$ & 55 & $3.413439798966(+1)$ & 101 & $2.044404857517(+1)$ \\
\hline 10 & $1.805201856422(+2)$ & 56 & $3.357454812058(+1)$ & 102 & $2.029805069664(+1)$ \\
\hline 11 & $1.641556933857(+2)$ & 57 & $3.303528133374(+1)$ & 103 & $2.015557079718(+1)$ \\
\hline 12 & $1.505226802369(+2)$ & 58 & $3.251553641437(+1)$ & 104 & $2.001651339383(+1)$ \\
\hline 13 & $1.389908073897(+2)$ & 59 & $3.201432418162(+1)$ & 105 & $1.988078679875(+1)$ \\
\hline 14 & $1.291098330474(+2)$ & 60 & $3.153072148750(+1)$ & 106 & $1.974830294518(+1)$ \\
\hline 15 & $1.205495800880(+2)$ & 61 & $3.106386580602(+1)$ & 107 & $1.961897722333(+1)$ \\
\hline 16 & $1.130624158535(+2)$ & 62 & $3.061295034613(+1)$ & 108 & $1.949272832559(+1)$ \\
\hline 17 & $1.064589743680(+2)$ & 63 & $3.017721963011(+1)$ & 109 & $1.936947810045(+1)$ \\
\hline 18 & $1.005919711489(+2)$ & 64 & $2.975596548653(+1)$ & 110 & $1.924915141466(+1)$ \\
\hline 19 & $9.534512913077(+1)$ & 65 & $2.934852341319(+1)$ & 111 & $1.913167602301(+1)$ \\
\hline 20 & $9.062542681220(+1)$ & 66 & $2.895426927075(+1)$ & 112 & $1.901698244536(+1)$ \\
\hline 21 & $8.635756121769(+1)$ & 67 & $2.857261627251(+1)$ & 113 & $1.890500385045(+1)$ \\
\hline 22 & $8.247992096101(+1)$ & 68 & $2.820301223984(+1)$ & 114 & $1.879567594604(+1)$ \\
\hline 23 & $7.894160981382(+1)$ & 69 & $2.784493709640(+1)$ & 115 & $1.868893687509(+1)$ \\
\hline 24 & $7.570021438219(+1)$ & 70 & $2.749790057713(+1)$ & 116 & $1.858472711757(+1)$ \\
\hline 25 & $7.272010754079(+1)$ & 71 & $2.716144013099(+1)$ & 117 & $1.848298939758(+1)$ \\
\hline 26 & $6.997114338234(+1)$ & 72 & $2.683511899850(+1)$ & 118 & $1.838366859551(+1)$ \\
\hline 27 & $6.742764217836(+1)$ & 73 & $2.651852444735(+1)$ & 119 & $1.828671166493(+1)$ \\
\hline 28 & $6.506759284831(+1)$ & 74 & $2.621126615118(+1)$ & 120 & $1.819206755398(+1)$ \\
\hline 29 & $6.287202043529(+1)$ & 75 & $2.591297469812(+1)$ & 121 & $1.809968713096(+1)$ \\
\hline 30 & $6.082448008652(+1)$ & 76 & $2.562330021713(+1)$ & 122 & $1.800952311396(+1)$ \\
\hline 31 & $5.891064897319(+1)$ & 77 & $2.534191111152(+1)$ & 123 & $1.792153000426(+1)$ \\
\hline 32 & $5.711799472544(+1)$ & 78 & $2.506849288987(+1)$ & 124 & $1.783566402340(+1)$ \\
\hline 33 & $5.543550415181(+1)$ & 79 & $2.480274708597(+1)$ & 125 & $1.775188305360(+1)$ \\
\hline 34 & $5.385345983192(+1)$ & 80 & $2.454439025968(+1)$ & 126 & $1.767014658145(+1)$ \\
\hline 35 & $5.236325500747(+1)$ & 81 & $2.429315307204(+1)$ & 127 & $1.759041564473(+1)$ \\
\hline 36 & $5.095723932487(+1)$ & 82 & $2.404877942805(+1)$ & 128 & $1.751265278217(+1)$ \\
\hline 37 & $4.962858959259(+1)$ & 83 & $2.381102568155(+1)$ & 129 & $1.743682198598(+1)$ \\
\hline 38 & $4.837120094531(+1)$ & 84 & $2.357965989698(+1)$ & 130 & $1.736288865710(+1)$ \\
\hline 39 & $4.717959475207(+1)$ & 85 & $2.335446116333(+1)$ & 131 & $1.729081956308(+1)$ \\
\hline 40 & $4.604884033827(+1)$ & 86 & $2.313521895613(+1)$ & 132 & $1.722058279828(+1)$ \\
\hline 41 & $4.497448816301(+1)$ & 87 & $2.292173254342(+1)$ & 133 & $1.715214774659(+1)$ \\
\hline 42 & $4.395251254260(+1)$ & 88 & $2.271381043244(+1)$ & 134 & $1.708548504632(+1)$ \\
\hline 43 & $4.297926236616(+1)$ & 89 & $2.251126985368(+1)$ & 135 & $1.702056655731(+1)$ \\
\hline 44 & $4.205141853187(+1)$ & 90 & $2.231393627942(+1)$ & 136 & $1.695736533017(+1)$ \\
\hline 45 & $4.116595705847(+1)$ & 91 & $2.212164297423(+1)$ & 137 & $1.689585557753(+1)$ \\
\hline 46 & $4.032011700828(+1)$ & 92 & $2.193423057473(+1)$ & & \\
\hline
\end{tabular}


Table 11

Magnetic shielding constants $\sigma$ (in units of $\left.\alpha^{2}\right)$ of Dirac one-electron atoms in the excited state $3 \mathrm{~d}_{3 / 2}(\mu= \pm 3 / 2)$. The number in brackets is the power of 10 by which the corresponding entry is to be multiplied. The value of the inverse of the fine-structure constant used was $\alpha^{-1}=137.035999139$ (from CODATA 2014).

\begin{tabular}{|c|c|c|c|c|c|}
\hline$Z$ & $\sigma\left[\alpha^{2}\right]$ & $Z$ & $\sigma\left[\alpha^{2}\right]$ & $Z$ & $\sigma\left[\alpha^{2}\right]$ \\
\hline 1 & $1.201888253354(+3)$ & 47 & $2.756044695895(+1)$ & 93 & $1.732057778713(+1)$ \\
\hline 2 & $6.010054646848(+2)$ & 48 & $2.707313019753(+1)$ & 94 & $1.724582439587(+1)$ \\
\hline 3 & $4.007384714441(+2)$ & 49 & $2.660776483764(+1)$ & 95 & $1.717458526708(+1)$ \\
\hline 4 & $3.006254364150(+2)$ & 50 & $2.616305183525(+1)$ & 96 & $1.710678455982(+1)$ \\
\hline 5 & $2.405739951535(+2)$ & 51 & $2.573779456173(+1)$ & 97 & $1.704235054005(+1)$ \\
\hline 6 & $2.005533620160(+2)$ & 52 & $2.533088896773(+1)$ & 98 & $1.698121540307(+1)$ \\
\hline 7 & $1.719789167354(+2)$ & 53 & $2.494131486101(+1)$ & 99 & $1.692331510790(+1)$ \\
\hline 8 & $1.505583510670(+2)$ & 54 & $2.456812815372(+1)$ & 100 & $1.686858922295(+1)$ \\
\hline 9 & $1.339070510039(+2)$ & 55 & $2.421045395594(+1)$ & 101 & $1.681698078219(+1)$ \\
\hline 10 & $1.205942496647(+2)$ & 56 & $2.386748040959(+1)$ & 102 & $1.676843615111(+1)$ \\
\hline 11 & $1.097094604245(+2)$ & 57 & $2.353845317178(+1)$ & 103 & $1.672290490205(+1)$ \\
\hline 12 & $1.006456934815(+2)$ & 58 & $2.322267046939(+1)$ & 104 & $1.668033969813(+1)$ \\
\hline 13 & $9.298272631635(+1)$ & 59 & $2.291947865689(+1)$ & 105 & $1.664069618536(+1)$ \\
\hline 14 & $8.642040109841(+1)$ & 60 & $2.262826821895(+1)$ & 106 & $1.660393289254(+1)$ \\
\hline 15 & $8.073860312973(+1)$ & 61 & $2.234847016658(+1)$ & 107 & $1.657001113834(+1)$ \\
\hline 16 & $7.577224737343(+1)$ & 62 & $2.207955278260(+1)$ & 108 & $1.653889494537(+1)$ \\
\hline 17 & $7.139509326648(+1)$ & 63 & $2.182101867747(+1)$ & 109 & $1.651055096069(+1)$ \\
\hline 18 & $6.750895459555(+1)$ & 64 & $2.157240212165(+1)$ & 110 & $1.648494838258(+1)$ \\
\hline 19 & $6.403631678089(+1)$ & 65 & $2.133326662469(+1)$ & 111 & $1.646205889319(+1)$ \\
\hline 20 & $6.091516897556(+1)$ & 66 & $2.110320273493(+1)$ & 112 & $1.644185659678(+1)$ \\
\hline 21 & $5.809531270816(+1)$ & 67 & $2.088182603672(+1)$ & 113 & $1.642431796340(+1)$ \\
\hline 22 & $5.553567725985(+1)$ & 68 & $2.066877532491(+1)$ & 114 & $1.640942177763(+1)$ \\
\hline 23 & $5.320233537818(+1)$ & 69 & $2.046371093862(+1)$ & 115 & $1.639714909234(+1)$ \\
\hline 24 & $5.106701506295(+1)$ & 70 & $2.026631323834(+1)$ & 116 & $1.638748318713(+1)$ \\
\hline 25 & $4.910596852378(+1)$ & 71 & $2.007628121236(+1)$ & 117 & $1.638040953141(+1)$ \\
\hline 26 & $4.729910214796(+1)$ & 72 & $1.989333119984(+1)$ & 118 & $1.637591575187(+1)$ \\
\hline 27 & $4.562929980915(+1)$ & 73 & $1.971719571949(+1)$ & 119 & $1.637399160432(+1)$ \\
\hline 28 & $4.408189118171(+1)$ & 74 & $1.954762239372(+1)$ & 120 & $1.637462894962(+1)$ \\
\hline 29 & $4.264423005941(+1)$ & 75 & $1.938437295961(+1)$ & 121 & $1.637782173380(+1)$ \\
\hline 30 & $4.130535701072(+1)$ & 76 & $1.922722235851(+1)$ & 122 & $1.638356597210(+1)$ \\
\hline 31 & $4.005572732701(+1)$ & 77 & $1.907595789727(+1)$ & 123 & $1.639185973703(+1)$ \\
\hline 32 & $3.888698998090(+1)$ & 78 & $1.893037847459(+1)$ & 124 & $1.640270315020(+1)$ \\
\hline 33 & $3.779180677424(+1)$ & 79 & $1.879029386696(+1)$ & 125 & $1.641609837808(+1)$ \\
\hline 34 & $3.676370340168(+1)$ & 80 & $1.865552406869(+1)$ & 126 & $1.643204963148(+1)$ \\
\hline 35 & $3.579694604642(+1)$ & 81 & $1.852589868167(+1)$ & 127 & $1.645056316888(+1)$ \\
\hline 36 & $3.488643854377(+1)$ & 82 & $1.840125635048(+1)$ & 128 & $1.647164730350(+1)$ \\
\hline 37 & $3.402763622127(+1)$ & 83 & $1.828144423912(+1)$ & 129 & $1.649531241414(+1)$ \\
\hline 38 & $3.321647334332(+1)$ & 84 & $1.816631754587(+1)$ & 130 & $1.652157095989(+1)$ \\
\hline 39 & $3.244930171853(+1)$ & 85 & $1.805573905323(+1)$ & 131 & $1.655043749863(+1)$ \\
\hline 40 & $3.172283851642(+1)$ & 86 & $1.794957871011(+1)$ & 132 & $1.658192870938(+1)$ \\
\hline 41 & $3.103412172093(+1)$ & 87 & $1.784771324366(+1)$ & 133 & $1.661606341872(+1)$ \\
\hline 42 & $3.038047194820(+1)$ & 88 & $1.775002579847(+1)$ & 134 & $1.665286263108(+1)$ \\
\hline 43 & $2.975945959241(+1)$ & 89 & $1.765640560099(+1)$ & 135 & $1.669234956321(+1)$ \\
\hline 44 & $2.916887645210(+1)$ & 90 & $1.756674764728(+1)$ & 136 & $1.673454968286(+1)$ \\
\hline 45 & $2.860671114005(+1)$ & 91 & $1.748095241232(+1)$ & 137 & $1.677949075176(+1)$ \\
\hline 46 & $2.807112770093(+1)$ & 92 & $1.739892557925(+1)$ & & \\
\hline
\end{tabular}


Table 12

Magnetic shielding constants $\sigma$ (in units of $\alpha^{2}$ ) of Dirac one-electron atoms in the excited state $3 \mathrm{~d}_{5 / 2}(\mu= \pm 1 / 2)$. The number in brackets is the power of 10 by which the corresponding entry is to be multiplied. The value of the inverse of the fine-structure constant used was $\alpha^{-1}=137.035999139$ (from CODATA 2014).

\begin{tabular}{|c|c|c|c|c|c|}
\hline$Z$ & $\sigma\left[\alpha^{2}\right]$ & $Z$ & $\sigma\left[\alpha^{2}\right]$ & $Z$ & $\sigma\left[\alpha^{2}\right]$ \\
\hline 1 & $-1.802737140970(+3)$ & 47 & $-3.676015209631(+1)$ & 93 & $-1.620610226415(+1)$ \\
\hline 2 & $-9.013177131173(+2)$ & 48 & $-3.592693915741(+1)$ & 94 & $-1.596512048885(+1)$ \\
\hline 3 & $-6.008219668249(+2)$ & 49 & $-3.512633354025(+1)$ & 95 & $-1.572846006106(+1)$ \\
\hline 4 & $-4.505571404725(+2)$ & 50 & $-3.435637805826(+1)$ & 96 & $-1.549598501840(+1)$ \\
\hline 5 & $-3.603846815919(+2)$ & 51 & $-3.361526901246(+1)$ & 97 & $-1.526756499035(+1)$ \\
\hline 6 & $-3.002584059068(+2)$ & 52 & $-3.290134143292(+1)$ & 98 & $-1.504307491265(+1)$ \\
\hline 7 & $-2.573013771957(+2)$ & 53 & $-3.221305599098(+1)$ & 99 & $-1.482239475915(+1)$ \\
\hline 8 & $-2.250751272575(+2)$ & 54 & $-3.154898736566(+1)$ & 100 & $-1.460540928970(+1)$ \\
\hline 9 & $-2.000027292335(+2)$ & 55 & $-3.090781387917(+1)$ & 101 & $-1.439200781302(+1)$ \\
\hline 10 & $-1.799380269362(+2)$ & 56 & $-3.028830824296(+1)$ & 102 & $-1.418208396347(+1)$ \\
\hline 11 & $-1.635152845438(+2)$ & 57 & $-2.968932927775(+1)$ & 103 & $-1.397553549087(+1)$ \\
\hline 12 & $-1.498240114481(+2)$ & 58 & $-2.910981449026(+1)$ & 104 & $-1.377226406247(+1)$ \\
\hline 13 & $-1.382338679411(+2)$ & 59 & $-2.854877340472(+1)$ & 105 & $-1.357217507612(+1)$ \\
\hline 14 & $-1.282946113223(+2)$ & 60 & $-2.800528156125(+1)$ & 106 & $-1.337517748410(+1)$ \\
\hline 15 & $-1.196760635625(+2)$ & 61 & $-2.747847510462(+1)$ & 107 & $-1.318118362686(+1)$ \\
\hline 16 & $-1.121305910943(+2)$ & 62 & $-2.696754589661(+1)$ & 108 & $-1.299010907583(+1)$ \\
\hline 17 & $-1.054688270285(+2)$ & 63 & $-2.647173709388(+1)$ & 109 & $-1.280187248508(+1)$ \\
\hline 18 & $-9.954348596623(+1)$ & 64 & $-2.599033914044(+1)$ & 110 & $-1.261639545090(+1)$ \\
\hline 19 & $-9.423828992202(+1)$ & 65 & $-2.552268612994(+1)$ & 111 & $-1.243360237908(+1)$ \\
\hline 20 & $-8.946021647054(+1)$ & 66 & $-2.506815249884(+1)$ & 112 & $-1.225342035922(+1)$ \\
\hline 21 & $-8.513396170829(+1)$ & 67 & $-2.462615001549(+1)$ & 113 & $-1.207577904574(+1)$ \\
\hline 22 & $-8.119791331672(+1)$ & 68 & $-2.419612503508(+1)$ & 114 & $-1.190061054518(+1)$ \\
\hline 23 & $-7.760117413061(+1)$ & 69 & $-2.377755599312(+1)$ & 115 & $-1.172784930927(+1)$ \\
\hline 24 & $-7.430132981435(+1)$ & 70 & $-2.336995111389(+1)$ & 116 & $-1.155743203368(+1)$ \\
\hline 25 & $-7.126275229591(+1)$ & 71 & $-2.297284631246(+1)$ & 117 & $-1.138929756179(+1)$ \\
\hline 26 & $-6.845529471600(+1)$ & 72 & $-2.258580327 \quad 156(+1)$ & 118 & $-1.122338679350(+1)$ \\
\hline 27 & $-6.585327638862(+1)$ & 73 & $-2.220840767654(+1)$ & 119 & $-1.105964259852(+1)$ \\
\hline 28 & $-6.343468526993(+1)$ & 74 & $-2.184026759336(+1)$ & 120 & $-1.089800973403(+1)$ \\
\hline 29 & $-6.118054543367(+1)$ & 75 & $-2.148101197645(+1)$ & 121 & $-1.073843476646(+1)$ \\
\hline 30 & $-5.907441105145(+1)$ & 76 & $-2.113028929426(+1)$ & 122 & $-1.058086599700(+1)$ \\
\hline 31 & $-5.710195831228(+1)$ & 77 & $-2.078776626198(+1)$ & 123 & $-1.042525339088(+1)$ \\
\hline 32 & $-5.525065385728(+1)$ & 78 & $-2.045312667171(+1)$ & 124 & $-1.027154850996(+1)$ \\
\hline 33 & $-5.350948349893(+1)$ & 79 & $-2.012607031147(+1)$ & 125 & $-1.011970444857(+1)$ \\
\hline 34 & $-5.186872881337(+1)$ & 80 & $-1.980631196527(+1)$ & 126 & $-9.969675772444(+0)$ \\
\hline 35 & $-5.031978203121(+1)$ & 81 & $-1.949358048730(+1)$ & 127 & $-9.821418460475(+0)$ \\
\hline 36 & $-4.885499177983(+1)$ & 82 & $-1.918761794381(+1)$ & 128 & $-9.674889849229(+0)$ \\
\hline 37 & $-4.746753384046(+1)$ & 83 & $-1.888817881700(+1)$ & 129 & $-9.530048580026(+0)$ \\
\hline 38 & $-4.615130231202(+1)$ & 84 & $-1.859502926584(+1)$ & 130 & $-9.386854548451(+0)$ \\
\hline 39 & $-4.490081751897(+1)$ & 85 & $-1.830794643901(+1)$ & 131 & $-9.245268856172(+0)$ \\
\hline 40 & $-4.371114773302(+1)$ & 86 & $-1.802671783579(+1)$ & 132 & $-9.105253764951(+0)$ \\
\hline 41 & $-4.257784235015(+1)$ & 87 & $-1.775114071100(+1)$ & 133 & $-8.966772652705(+0)$ \\
\hline 42 & $-4.149687461377(+1)$ & 88 & $-1.748102152 \quad 057(+1)$ & 134 & $-8.829789971546(+0)$ \\
\hline 43 & $-4.046459233005(+1)$ & 89 & $-1.721617540438(+1)$ & 135 & $-8.694271207662(+0)$ \\
\hline 44 & $-3.947767530382(+1)$ & 90 & $-1.695642570370(+1)$ & 136 & $-8.560182842975(+0)$ \\
\hline 45 & $-3.853309844967(+1)$ & 91 & $-1.670160351032(+1)$ & 137 & $-8.427492318459(+0)$ \\
\hline 46 & $-3.762809971473(+1)$ & 92 & $-1.645154724519(+1)$ & & \\
\hline
\end{tabular}


Table 13

Magnetic shielding constants $\sigma$ (in units of $\left.\alpha^{2}\right)$ of Dirac one-electron atoms in the excited state $3 \mathrm{~d}_{5 / 2}(\mu= \pm 3 / 2)$. The number in brackets is the power of 10 by which the corresponding entry is to be multiplied. The value of the inverse of the fine-structure constant used was $\alpha^{-1}=137.035999139$ (from CODATA 2014).

\begin{tabular}{|c|c|c|c|c|c|}
\hline$Z$ & $\sigma\left[\alpha^{2}\right]$ & $Z$ & $\sigma\left[\alpha^{2}\right]$ & $Z$ & $\sigma\left[\alpha^{2}\right]$ \\
\hline 1 & $-1.201808887437(+3)$ & 47 & $-2.374033490069(+1)$ & 93 & $-9.162820482311(+0)$ \\
\hline 2 & $-6.008467278303(+2)$ & 48 & $-2.316764285622(+1)$ & 94 & $-8.980691814721(+0)$ \\
\hline 3 & $-4.005003536060(+2)$ & 49 & $-2.261663037289(+1)$ & 95 & $-8.801311165362(+0)$ \\
\hline 4 & $-3.003079225213(+2)$ & 50 & $-2.208599131022(+1)$ & 96 & $-8.624585901323(+0)$ \\
\hline 5 & $-2.401770651011(+2)$ & 51 & $-2.157452184453(+1)$ & 97 & $-8.450427096045(+0)$ \\
\hline 6 & $-2.000769906643(+2)$ & 52 & $-2.108111062974(+1)$ & 98 & $-8.278749338578(+0)$ \\
\hline 7 & $-1.714230738988(+2)$ & 53 & $-2.060473007195(+1)$ & 99 & $-8.109470554358(+0)$ \\
\hline 8 & $-1.499230015052(+2)$ & 54 & $-2.014442857353(+1)$ & 100 & $-7.942511836706(+0)$ \\
\hline 9 & $-1.331921544114(+2)$ & 55 & $-1.969932362323(+1)$ & 101 & $-7.777797288294(+0)$ \\
\hline 10 & $-1.197997606581(+2)$ & 56 & $-1.926859562665(+1)$ & 102 & $-7.615253871906(+0)$ \\
\hline 11 & $-1.088353285285(+2)$ & 57 & $-1.885148238610(+1)$ & 103 & $-7.454811269849(+0)$ \\
\hline 12 & $-9.969186311332(+1)$ & 58 & $-1.844727415144(+1)$ & 104 & $-7.296401751444(+0)$ \\
\hline 13 & $-9.194913676850(+1)$ & 59 & $-1.805530917429(+1)$ & 105 & $-7.139960048044(+0)$ \\
\hline 14 & $-8.530698651973(+1)$ & 60 & $-1.767496970665(+1)$ & 106 & $-6.985423235088(+0)$ \\
\hline 15 & $-7.954529250499(+1)$ & 61 & $-1.730567839323(+1)$ & 107 & $-6.832730620735(+0)$ \\
\hline 16 & $-7.449896450116(+1)$ & 62 & $-1.694689501276(+1)$ & 108 & $-6.681823640642(+0)$ \\
\hline 17 & $-7.004175673523(+1)$ & 63 & $-1.659811352967(+1)$ & 109 & $-6.532645758489(+0)$ \\
\hline 18 & $-6.607547775840(+1)$ & 64 & $-1.625885942216(+1)$ & 110 & $-6.385142371894(+0)$ \\
\hline 19 & $-6.252260772833(+1)$ & 65 & $-1.592868725685(+1)$ & 111 & $-6.239260723373(+0)$ \\
\hline 20 & $-5.932113050662(+1)$ & 66 & $-1.560717848392(+1)$ & 112 & $-6.094949816020(+0)$ \\
\hline 21 & $-5.642084229988(+1)$ & 67 & $-1.529393942967(+1)$ & 113 & $-5.952160333632(+0)$ \\
\hline 22 & $-5.378066703492(+1)$ & 68 & $-1.498859946612(+1)$ & 114 & $-5.810844564984(+0)$ \\
\hline 23 & $-5.136667207088(+1)$ & 69 & $-1.469080933984(+1)$ & 115 & $-5.670956332020(+0)$ \\
\hline 24 & $-4.915057998323(+1)$ & 70 & $-1.440023964394(+1)$ & 116 & $-5.532450921704(+0)$ \\
\hline 25 & $-4.710863751961(+1)$ & 71 & $-1.411657941914(+1)$ & 117 & $-5.395285021325(+0)$ \\
\hline 26 & $-4.522074556578(+1)$ & 72 & $-1.383953487146(+1)$ & 118 & $-5.259416657050(+0)$ \\
\hline 27 & $-4.346978245245(+1)$ & 73 & $-1.356882819521(+1)$ & 119 & $-5.124805135516(+0)$ \\
\hline 28 & $-4.184107226774(+1)$ & 74 & $-1.330419649141(+1)$ & 120 & $-4.991410988308(+0)$ \\
\hline 29 & $-4.032196317395(+1)$ & 75 & $-1.304539077271(+1)$ & 121 & $-4.859195919130(+0)$ \\
\hline 30 & $-3.890149006087(+1)$ & 76 & $-1.279217504684(+1)$ & 122 & $-4.728122753535(+0)$ \\
\hline 31 & $-3.757010249208(+1)$ & 77 & $-1.254432547144(+1)$ & 123 & $-4.598155391044(+0)$ \\
\hline 32 & $-3.631944366111(+1)$ & 78 & $-1.230162957391(+1)$ & 124 & $-4.469258759549(+0)$ \\
\hline 33 & $-3.514216953756(+1)$ & 79 & $-1.206388553040(+1)$ & 125 & $-4.341398771832(+0)$ \\
\hline 34 & $-3.403179992837(+1)$ & 80 & $-1.183090149894(+1)$ & 126 & $-4.214542284120(+0)$ \\
\hline 35 & $-3.298259507160(+1)$ & 81 & $-1.160249500189(+1)$ & 127 & $-4.088657056531(+0)$ \\
\hline 36 & $-3.198945279771(+1)$ & 82 & $-1.137849235356(+1)$ & 128 & $-3.963711715329(+0)$ \\
\hline 37 & $-3.104782236751(+1)$ & 83 & $-1.115872812915(+1)$ & 129 & $-3.839675716869(+0)$ \\
\hline 38 & $-3.015363191447(+1)$ & 84 & $-1.094304467162(+1)$ & 130 & $-3.716519313161(+0)$ \\
\hline 39 & $-2.930322704985(+1)$ & 85 & $-1.073129163334(+1)$ & 131 & $-3.594213518945(+0)$ \\
\hline 40 & $-2.849331867693(+1)$ & 86 & $-1.052332554969(+1)$ & 132 & $-3.472730080206(+0)$ \\
\hline 41 & $-2.772093844220(+1)$ & 87 & $-1.031900944197(+1)$ & 133 & $-3.352041444053(+0)$ \\
\hline 42 & $-2.698340055062(+1)$ & 88 & $-1.011821244748(+1)$ & 134 & $-3.232120729879(+0)$ \\
\hline 43 & $-2.627826890899(+1)$ & 89 & $-9.920809474378(+0)$ & 135 & $-3.112941701750(+0)$ \\
\hline 44 & $-2.560332874964(+1)$ & 90 & $-9.726680879577(+0)$ & 136 & $-2.994478741939(+0)$ \\
\hline 45 & $-2.495656203775(+1)$ & 91 & $-9.535712167860(+0)$ & 137 & $-2.876706825563(+0)$ \\
\hline 46 & $-2.433612608623(+1)$ & 92 & $-9.347793710550(+0)$ & & \\
\hline
\end{tabular}


Table 14

Magnetic shielding constants $\sigma$ (in units of $\alpha^{2}$ ) of Dirac one-electron atoms in the excited state $3 \mathrm{~d}_{3 / 2}(\mu= \pm 5 / 2)$. The number in brackets is the power of 10 by which the corresponding entry is to be multiplied. The value of the inverse of the fine-structure constant used was $\alpha^{-1}=137.035999139$ (from CODATA 2014).

\begin{tabular}{|c|c|c|c|c|c|}
\hline$Z$ & $\sigma\left[\alpha^{2}\right]$ & $Z$ & $\sigma\left[\alpha^{2}\right]$ & $Z$ & $\sigma\left[\alpha^{2}\right]$ \\
\hline 1 & $4.761962866692(-2)$ & 47 & $2.299299490566(+0)$ & 93 & $4.923743081357(+0)$ \\
\hline 2 & $9.524274371172(-2)$ & 48 & $2.350949746169(+0)$ & 94 & $4.988165533544(+0)$ \\
\hline 3 & $1.428728319655(-1)$ & 49 & $2.402775961817(+0)$ & 95 & $5.052986626024(+0)$ \\
\hline 4 & $1.905133811660(-1)$ & 50 & $2.454782185846(+0)$ & 96 & $5.118212332840(+0)$ \\
\hline 5 & $2.381678804110(-1)$ & 51 & $2.506972491314(+0)$ & 97 & $5.183848692567(+0)$ \\
\hline 6 & $2.858398206127(-1)$ & 52 & $2.559350976615(+0)$ & 98 & $5.249901809561(+0)$ \\
\hline 7 & $3.335326949516(-1)$ & 53 & $2.611921766103(+0)$ & 99 & $5.316377855222(+0)$ \\
\hline 8 & $3.812499993322(-1)$ & 54 & $2.664689010726(+0)$ & 100 & $5.383283069291(+0)$ \\
\hline 9 & $4.289952328380(-1)$ & 55 & $2.717656888659(+0)$ & 101 & $5.450623761161(+0)$ \\
\hline 10 & $4.767718981891(-1)$ & 56 & $2.770829605958(+0)$ & 102 & $5.518406311217(+0)$ \\
\hline 11 & $5.245835021988(-1)$ & 57 & $2.824211397210(+0)$ & 103 & $5.586637172202(+0)$ \\
\hline 12 & $5.724335562331(-1)$ & 58 & $2.877806526199(+0)$ & 104 & $5.655322870609(+0)$ \\
\hline 13 & $6.203255766703(-1)$ & 59 & $2.931619286576(+0)$ & 105 & $5.724470008100(+0)$ \\
\hline 14 & $6.682630853624(-1)$ & 60 & $2.985654002542(+0)$ & 106 & $5.794085262946(+0)$ \\
\hline 15 & $7.162496100974(-1)$ & 61 & $3.039915029535(+0)$ & 107 & $5.864175391509(+0)$ \\
\hline 16 & $7.642886850646(-1)$ & 62 & $3.094406754934(+0)$ & 108 & $5.934747229734(+0)$ \\
\hline 17 & $8.123838513195(-1)$ & 63 & $3.149133598760(+0)$ & 109 & $6.005807694687(+0)$ \\
\hline 18 & $8.605386572527(-1)$ & 64 & $3.204100014404(+0)$ & 110 & $6.077363786116(+0)$ \\
\hline 19 & $9.087566590593(-1)$ & 65 & $3.259310489347(+0)$ & 111 & $6.149422588039(+0)$ \\
\hline 20 & $9.570414212110(-1)$ & 66 & $3.314769545904(+0)$ & 112 & $6.221991270374(+0)$ \\
\hline 21 & $1.005396516930(+0)$ & 67 & $3.370481741971(+0)$ & 113 & $6.295077090589(+0)$ \\
\hline 22 & $1.053825528668(+0)$ & 68 & $3.426451671785(+0)$ & 114 & $6.368687395398(+0)$ \\
\hline 23 & $1.102332048579(+0)$ & 69 & $3.482683966697(+0)$ & 115 & $6.442829622480(+0)$ \\
\hline 24 & $1.150919679010(+0)$ & 70 & $3.539183295953(+0)$ & 116 & $6.517511302241(+0)$ \\
\hline 25 & $1.199592032978(+0)$ & 71 & $3.595954367489(+0)$ & 117 & $6.592740059608(+0)$ \\
\hline 26 & $1.248352734661(+0)$ & 72 & $3.653001928737(+0)$ & 118 & $6.668523615859(+0)$ \\
\hline 27 & $1.297205419889(+0)$ & 73 & $3.710330767441(+0)$ & 119 & $6.744869790495(+0)$ \\
\hline 28 & $1.346153736633(+0)$ & 74 & $3.767945712494(+0)$ & 120 & $6.821786503146(+0)$ \\
\hline 29 & $1.395201345509(+0)$ & 75 & $3.825851634773(+0)$ & 121 & $6.899281775519(+0)$ \\
\hline 30 & $1.444351920274(+0)$ & 76 & $3.884053448007(+0)$ & 122 & $6.977363733389(+0)$ \\
\hline 31 & $1.493609148328(+0)$ & 77 & $3.942556109635(+0)$ & 123 & $7.056040608626(+0)$ \\
\hline 32 & $1.542976731227(+0)$ & 78 & $4.001364621701(+0)$ & 124 & $7.135320741268(+0)$ \\
\hline 33 & $1.592458385193(+0)$ & 79 & $4.060484031745(+0)$ & 125 & $7.215212581644(+0)$ \\
\hline 34 & $1.642057841625(+0)$ & 80 & $4.119919433722(+0)$ & 126 & $7.295724692528(+0)$ \\
\hline 35 & $1.691778847628(+0)$ & 81 & $4.179675968925(+0)$ & 127 & $7.376865751355(+0)$ \\
\hline 36 & $1.741625166527(+0)$ & 82 & $4.239758826929(+0)$ & 128 & $7.458644552472(+0)$ \\
\hline 37 & $1.791600578404(+0)$ & 83 & $4.300173246552(+0)$ & 129 & $7.541070009446(+0)$ \\
\hline 38 & $1.841708880627(+0)$ & 84 & $4.360924516828(+0)$ & 130 & $7.624151157418(+0)$ \\
\hline 39 & $1.891953888390(+0)$ & 85 & $4.422017977998(+0)$ & 131 & $7.707897155510(+0)$ \\
\hline 40 & $1.942339435254(+0)$ & 86 & $4.483459022519(+0)$ & 132 & $7.792317289283(+0)$ \\
\hline 41 & $1.992869373698(+0)$ & 87 & $4.545253096086(+0)$ & 133 & $7.877420973251(+0)$ \\
\hline 42 & $2.043547575672(+0)$ & 88 & $4.607405698681(+0)$ & 134 & $7.963217753453(+0)$ \\
\hline 43 & $2.094377933154(+0)$ & 89 & $4.669922385624(+0)$ & 135 & $8.049717310074(+0)$ \\
\hline 44 & $2.145364358717(+0)$ & 90 & $4.732808768664(+0)$ & 136 & $8.136929460133(+0)$ \\
\hline 45 & $2.196510786100(+0)$ & 91 & $4.796070517066(+0)$ & 137 & $8.224864160228(+0)$ \\
\hline 46 & $2.247821170782(+0)$ & 92 & $4.859713358737(+0)$ & & \\
\hline
\end{tabular}


Table 15

Comparison of present values (the upper entries) of the magnetic shielding constants $\sigma$ (in units of $\alpha^{2}$ ) for selected Dirac one-electron atoms in the states with zero radial quantum number, i.e: $1 \mathrm{~s}_{1 / 2}, 2 \mathrm{p}_{3 / 2}$, and $3 \mathrm{~d}_{5 / 2}$, with those reported by Moore [22] (the lower entries). All the numbers were obtained using $\alpha^{-1}=137.0359895$ (from CODATA 1986). The present results were computed from the analytical formula given by Eqs. (1)-(4).

\begin{tabular}{|c|c|c|c|c|c|c|}
\hline$Z$ & $1 s_{1 / 2}$ & $2 p_{3 / 2}(\mu= \pm 1 / 2)$ & $2 p_{3 / 2}(\mu= \pm 3 / 2)$ & $3 d_{5 / 2}(\mu= \pm 1 / 2)$ & $3 d_{5 / 2}(\mu= \pm 3 / 2)$ & $3 d_{5 / 2}(\mu= \pm 5 / 2)$ \\
\hline 1 & $\begin{array}{lllll}0.333 & 381 & 164 & 739 & 8 \\
0.333 & 381 & 19 & & \end{array}$ & $\begin{array}{l}-2781.96850604 \\
-2773.93465574\end{array}$ & $\begin{array}{l}0.1000028534297 \\
0.10000286\end{array}$ & $\begin{array}{l}-1802.736887360 \\
-1789.53846854\end{array}$ & $\begin{array}{l}-1201.808718363 \\
-1193.00977248\end{array}$ & $\begin{array}{l}0.047619628667 \\
0.04761963\end{array}$ \\
\hline 2 & $\begin{array}{l}0.6670494090106 \\
0.66704943\end{array}$ & $\begin{array}{l}-1390.856472495 \\
-1389.95635659\end{array}$ & $\begin{array}{l}0.2000228284983 \\
0.20002285\end{array}$ & $\begin{array}{l}-901.3175863119 \\
-899.21878323\end{array}$ & $\begin{array}{l}-600.8466432933 \\
-599.44744123\end{array}$ & $\begin{array}{l}0.09524274371237 \\
0.09524275\end{array}$ \\
\hline 3 & $\begin{array}{l}1.001292268112 \\
1.00129232\end{array}$ & $\begin{array}{l}-927.0956650823 \\
-927.2026167\end{array}$ & 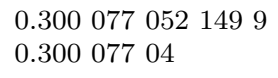 & $\begin{array}{l}-600.821882288 \\
-600.4330221\end{array}$ & $\begin{array}{l}-400.500297248 \\
-400.24106386\end{array}$ & 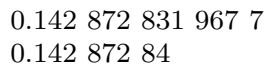 \\
\hline 4 & $\begin{array}{l}1.336398190326 \\
1.33639828\end{array}$ & $\begin{array}{l}-695.1726587054 \\
-695.13933063\end{array}$ & $\begin{array}{l}0.400182661942 \\
0.40018267\end{array}$ & $\begin{array}{l}-450.5570770698 \\
-450.64827181\end{array}$ & $\begin{array}{l}-300.3078802528 \\
-300.36867675\end{array}$ & $\begin{array}{l}0.1905133811712 \\
0.19051338\end{array}$ \\
\hline 5 & $\begin{array}{l}1.672656997017 \\
1.672656682\end{array}$ & $\begin{array}{l}-555.9847665823 \\
-556.01213569\end{array}$ & $\begin{array}{l}0.5003568113606 \\
0.50035679\end{array}$ & $\begin{array}{l}-360.3846308697 \\
-360.3534168\end{array}$ & $\begin{array}{l}-240.1770312864 \\
-240.1562219\end{array}$ & $\begin{array}{lllll}0.238 & 167 & 880 & 421 & 2 \\
0.238 & 167 & 89\end{array}$ \\
\hline 10 & $\begin{array}{l}3.381543230498 \\
3.38154202\end{array}$ & $\begin{array}{l}-277.353150433 \\
-277.75082306\end{array}$ & $\begin{array}{l}1.0028578121 \\
1.00285772\end{array}$ & $\begin{array}{l}-179.9380015751 \\
-179.94239823\end{array}$ & $\begin{array}{l}-119.7997437507 \\
-119.80267487\end{array}$ & $\begin{array}{lllll}0.476 & 771 & 898 & 270 & 9 \\
0.476 & 771 & 89\end{array}$ \\
\hline 20 & $\begin{array}{l}7.061805330068 \\
7.06179698\end{array}$ & $\begin{array}{l}-137.3960332144 \\
-137.39947467\end{array}$ & $\begin{array}{l}2.022969378746 \\
2.0229688\end{array}$ & $\begin{array}{l}-89.46020378996 \\
-89.46159879\end{array}$ & $\begin{array}{l}-59.32112205269 \\
-59.32205208\end{array}$ & $\begin{array}{l}0.9570414218684 \\
0.95704135\end{array}$ \\
\hline 50 & $\begin{array}{l}24.104073 \quad 17346 \\
24.10389279\end{array}$ & $\begin{array}{l}-51.33432147244 \\
-51.33549685\end{array}$ & $\begin{array}{l}5.371024990461 \\
5.37101684\end{array}$ & $\begin{array}{l}-34.35637298550 \\
-34.35693803\end{array}$ & $\begin{array}{l}-22.08598792486 \\
-22.08636501\end{array}$ & $\begin{array}{l}2.454782196404 \\
2.45478102\end{array}$ \\
\hline
\end{tabular}

\section{Table 16}

Comparison of present values (the upper entries) of the magnetic shielding constants $\sigma$ (in units of $10^{-6}$ ) for selected Dirac one-electron atoms in the ground state $\left(\mathrm{s}_{1 / 2}\right)$ and the first excited state, i.e: $2 \mathrm{p}_{1 / 2}$ and $2 \mathrm{p}_{3 / 2}$, with those obtained by Pyper and Zhang [23] (the lower entries). All the numbers were obtained using $\alpha^{-1}=137.0359895$ (from CODATA 1986). The present results were computed from the analytical formula given by Eqs. (1)-(4).

\begin{tabular}{|c|c|c|c|c|}
\hline$Z$ & $1 s_{1 / 2}$ & $2 p_{1 / 2}$ & $2 p_{3 / 2}(\mu= \pm 1 / 2)$ & $2 p_{3 / 2}(\mu= \pm 3 / 2)$ \\
\hline 1 & $\begin{array}{l}17.75300107721 \\
17.8\end{array}$ & $\begin{array}{l}148151.5997747 \\
148151.6\end{array}$ & $\begin{array}{l}-148143.6119015 \\
-148143.6\end{array}$ & $\begin{array}{llll}5.325 & 288 & 145917 \\
5.3 & & \end{array}$ \\
\hline 11 & $\begin{array}{l}198.6777362353 \\
198.7\end{array}$ & $\begin{array}{l}13506.18045156 \\
13506.2\end{array}$ & $\begin{array}{l}-13418.08895859 \\
-13418.1\end{array}$ & $\begin{array}{l}58.77911892966 \\
58.8\end{array}$ \\
\hline 19 & $\begin{array}{l}355.2419517328 \\
355.23\end{array}$ & $\begin{array}{c}7863.9041986173 \\
-\end{array}$ & $\begin{array}{c}-7710.94813558 \\
-\end{array}$ & $\begin{array}{c}102.2256496906 \\
-\end{array}$ \\
\hline 37 & $\begin{array}{l}801.3899985484 \\
801.4\end{array}$ & $\begin{array}{l}4140.214959702 \\
4140.2\end{array}$ & $\begin{array}{l}-3835.155688112 \\
-3835.2\end{array}$ & $\begin{array}{l}204.8932385444 \\
204.9\end{array}$ \\
\hline 55 & $\begin{array}{l}1531.456664843 \\
1531.4\end{array}$ & $\begin{array}{l}2916.272169119 \\
2916.3\end{array}$ & $\begin{array}{l}-2440.717541381 \\
-2440.7\end{array}$ & $\begin{array}{l}319.4036250787 \\
319.4\end{array}$ \\
\hline 74 & $\begin{array}{l}3102.065446132 \\
3101.9\end{array}$ & $\begin{array}{l}2364.616076795 \\
2364.6\end{array}$ & $\begin{array}{l}-1657.742849145 \\
-1657.7\end{array}$ & $\begin{array}{l}461.323573277 \\
461.3\end{array}$ \\
\hline 92 & $\begin{array}{l}7151.453606650 \\
7151.0\end{array}$ & $\begin{array}{l}2268.098559174 \\
2268.1\end{array}$ & $\begin{array}{l}-1175.683396542 \\
-1175.7\end{array}$ & $\begin{array}{l}626.0024046457 \\
626.0\end{array}$ \\
\hline
\end{tabular}

